\title{
Stability estimates for non-local scalar conservation laws
}

\author{
Felisia Angela Chiarello ${ }^{1} \quad$ Paola Goatin $^{1} \quad$ Elena Rossi $^{1}$
}

\begin{abstract}
We prove the stability of entropy weak solutions of a class of scalar conservation laws with non-local flux arising in traffic modelling. We obtain an estimate of the dependence of the solution with respect to the kernel function, the speed and the initial datum. Stability is obtained from the entropy condition through doubling of variable technique. We finally provide some numerical simulations illustrating the dependencies above for some cost functionals derived from traffic flow applications.
\end{abstract}

2010 Mathematics Subject Classification: 35L65, 35L60, 35L04, 90B20

Key words: Scalar conservation laws; Non-local flux; Stability.

\section{Introduction}

Conservation laws with non-local flux have drawn growing attention in the recent years. Indeed, beside the intrinsic mathematical interest for their properties, they turned out to be suitable for modelling several phenomena arising in natural or engineering sciences: flux functions depending on space-integrals of the unknown appear for example in models for granular flows [3, sedimentation [5], supply chains [15], conveyor belts [14], weakly coupled oscillators [2], structured populations dynamics [20] and traffic flows [6, 8, 21].

For this type of equations, general existence and uniqueness results have been established in [4, 7] for specific classes of scalar equations in one space-dimension, and in [1 for multidimensional systems of equations coupled through the non-local term. In particular, existence is usually proved by providing suitable compactness estimates on a sequence of approximate solutions constructed by finite volume schemes, while $\mathbf{L}^{\mathbf{1}}$-stability on initial data is obtained from Kružkov-type entropy conditions through the doubling of variable technique [18. A different approach based on fixed-point techniques has been recently proposed in [17] to prove existence and uniqueness of solutions to scalar balance laws in one space dimension, whose velocity term depends on the weighted integral of the density over an area in space.

In this paper, we focus on a specific class of scalar equations, in which the integral dependence of the flux function is expressed though a convolution product. We consider the following Cauchy problem

$$
\begin{cases}\partial_{t} \rho+\partial_{x}(f(t, x, \rho) V(t, x))=0 & t>0, x \in \mathbb{R}, \\ \rho(0, x)=\rho_{o}(x), & x \in \mathbb{R},\end{cases}
$$

\footnotetext{
${ }^{1}$ Inria Sophia Antipolis - Méditerranée, Université Côte d'Azur, Inria, CNRS, LJAD, 2004 route des Lucioles - BP 93, 06902 Sophia Antipolis Cedex, France. E-mail: ffelisia.chiarello, paola.goatin, elena.rossi\}@inria.fr
} 
where $V(t, x)=v((\rho(t) * w)(x))$, and $w$ is a smooth mollifier:

$$
(\rho(t) * w)(x)=\int_{\mathbb{R}} \rho(t, y) w(x-y) \mathrm{d} y .
$$

Here and below, we set $\rho(t):=\rho(t, \cdot)$ the function $x \mapsto \rho(t, x)$.

Existence and uniqueness of solutions to (1.1) follows from [4], as well as some a priori estimates, namely $\mathbf{L}^{\mathbf{1}}, \mathbf{L}^{\infty}$ and total variation estimates, see Section 2 below.

Motivated by the study of control and optimisation problems, we are interested in studying the dependence of solutions to (1.1) on the convolution kernel $w$ and on the velocity function $v$. Estimates of the dependence of solutions to a general balance laws on the flux function can be found in [11, 19]. However, as precised also below (see Remark 3), those estimates turn out to be implicit when applied to the setting of problem (1.1).

Carefully applying the Kružkov's doubling of variables techniques, on the lines of [5, 16, we derive the $\mathbf{L}^{1}$-Lipschitz continuous dependence of solutions to (1.1) on the initial datum, the kernel (see Theorem 11) and the velocity (see Theorem 2). These results are collected in Section 2, while the technical proofs are deferred to Section 3. Finally, in Section 4 we show some numerical simulation illustrating the behaviour of the solutions of a non-local traffic flow model, when the size and the position of the kernel support or the velocity function vary. In particular, we analyse the impact on two cost functionals, measuring traffic congestion.

\section{Main Results}

The study of problem (1.1) is carried out in the same setting of [4], with slightly strengthened conditions. We recall here briefly the assumptions on the flux function $f$, on $v$ and on $w$ :

$$
\begin{gathered}
f \in \mathbf{C}^{2}\left(\mathbb{R} \times \mathbb{R} \times \mathbb{R} ; \mathbb{R}^{+}\right) \text {and }\left\{\begin{array}{c}
\sup _{t, x, \rho}\left|\partial_{\rho} f(t, x, \rho)\right|<+\infty \\
\sup _{t, x}\left|\partial_{x} f(t, x, \rho)\right|<C|\rho| \\
\sup _{t, x}\left|\partial_{x x}^{2} f(t, x, \rho)\right|<C|\rho| \\
\forall t, x \quad f(t, x, 0)=0
\end{array}\right. \\
v \in\left(\mathbf{C}^{2} \cap \mathbf{W}^{2, \infty}\right)(\mathbb{R} ; \mathbb{R}) \text { and } w \in\left(\mathbf{C}^{2} \cap \mathbf{W}^{1,1} \cap \mathbf{W}^{2, \infty}\right)(\mathbb{R} ; \mathbb{R}) .
\end{gathered}
$$

We recall also the definition of solution to problem (1.1), see [4, Definition 2.1].

Definition 1. Let $T>0$. Fix $\rho_{o} \in \mathbf{L}^{\infty}(\mathbb{R} ; \mathbb{R})$. A weak entropy solution to (1.1) on $[0, T]$ is a bounded measurable Kružkov solution $\rho \in \mathbf{C}^{\mathbf{0}}\left([0, T] ; \mathbf{L}_{\text {loc }}^{1}(\mathbb{R} ; \mathbb{R})\right)$ to

$$
\left\{\begin{array}{l}
\partial_{t} \rho+\partial_{x}(f(t, x, \rho) V(t, x))=0 \\
\rho(0, x)=\rho_{o}(x)
\end{array} \quad \text { where } \quad V(t, x)=v((\rho(t) * w)(x)) .\right.
$$

The results in [4] ensure the existence and uniqueness of solution to (1.1) and provides the following a priori estimates on the solution.

Lemma 1 ([4, Lemma 2.2]). Let conditions (2.1)-(2.2) hold. If $\rho_{o}(x) \geq 0$ for all $x \in \mathbb{R}$, then the solution to (1.1) is such that $\rho(t, x) \geq 0$ for all $(t, x) \in \mathbb{R}^{+} \times \mathbb{R}$. 
Lemma 2 ([4, Lemma 2.4]). Let conditions (2.1)-(2.2) hold. If $\rho_{o}(x) \geq 0$ for all $x \in \mathbb{R}$, then the solution to (1.1) satisfies, for all $t \in \mathbb{R}^{+}$,

$$
\|\rho(t)\|_{\mathbf{L}^{1}(\mathbb{R} ; \mathbb{R})} \leq\left\|\rho_{o}\right\|_{\mathbf{L}^{1}(\mathbb{R} ; \mathbb{R})} .
$$

Lemma 3 ([4, Lemma 2.5]). Let conditions (2.1)-(2.2) hold. If $\rho_{o}(x) \geq 0$ for all $x \in \mathbb{R}$, then the solution to (1.1) satisfies, for all $t \in \mathbb{R}^{+}$,

$$
\|\rho(t)\|_{\mathbf{L}^{\infty}(\mathbb{R} ; \mathbb{R})} \leq\left\|\rho_{o}\right\|_{\mathbf{L}^{\infty}(\mathbb{R} ; \mathbb{R})} e^{\mathcal{L} t}
$$

where $\mathcal{L}=C\|v\|_{\mathbf{L}^{\infty}(\mathbb{R} ; \mathbb{R})}+\left\|\partial_{\rho} f\right\|_{\mathbf{L}^{\infty}([0, t] \times \mathbb{R} \times \mathbb{R} ; \mathbb{R})}\left\|v^{\prime}\right\|_{\mathbf{L}^{\infty}(\mathbb{R} ; \mathbb{R})}\left\|\rho_{o_{o}}\right\|_{\mathbf{L}^{\mathbf{1}(\mathbb{R} ; \mathbb{R})}}\left\|w^{\prime}\right\|_{\mathbf{L}^{\infty}(\mathbb{R} ; \mathbb{R})}$.

Proposition 1 (44, Proposition 2.6]). Let conditions (2.1) -(2.2) hold. If $\rho_{o}(x) \geq 0$ for all $x \in \mathbb{R}$, then the solution to (1.1) satisfies the following total variation estimate: for all $t \in \mathbb{R}^{+}$

$$
\mathrm{TV}(\rho(t)) \leq\left(\mathcal{K}_{2} t+\mathrm{TV}\left(\rho_{o}\right)\right) e^{\mathcal{K}_{1} t}
$$

where

$$
\begin{aligned}
\mathcal{K}_{1}= & \left\|\partial_{\rho x}^{2} f\right\|_{\mathbf{L}^{\infty}\left(\Sigma_{t} ; \mathbb{R}\right)}\|v\|_{\mathbf{L}^{\infty}(\mathbb{R} ; \mathbb{R})}, \\
\mathcal{K}_{2}= & {\left[\frac{3}{2} C+\left(\left\|\partial_{\rho} f\right\|_{\mathbf{L}^{\infty}\left(\Sigma_{t} ; \mathbb{R}\right)}+C\right)\left\|w^{\prime}\right\|_{\mathbf{W}^{1, \infty}(\mathbb{R} ; \mathbb{R})}\left\|\rho_{o}\right\|_{\mathbf{L}^{1}(\mathbb{R} ; \mathbb{R})}\right.} \\
& \left.+\frac{1}{2}\left(C+\left\|\partial_{\rho} f\right\|_{\mathbf{L}^{\infty}\left(\Sigma_{t} ; \mathbb{R}\right)}\left(2+\left\|\rho_{o}\right\|_{\mathbf{L}^{1}(\mathbb{R} ; \mathbb{R})}\left\|w^{\prime}\right\|_{\mathbf{L}^{\infty}(\mathbb{R} ; \mathbb{R})}\right)\right)\left\|w^{\prime}\right\|_{\mathbf{W}^{1, \infty}(\mathbb{R} ; \mathbb{R})}\right] \\
& \times\|v\|_{\mathbf{W}^{2, \infty}(\mathbb{R} ; \mathbb{R})}\left\|\rho_{o}\right\|_{\mathbf{L}^{1}(\mathbb{R} ; \mathbb{R})},
\end{aligned}
$$

with $\Sigma_{t}=[0, t] \times \mathbb{R} \times\left[0, M_{t}\right]$ and $M_{t}=\left\|\rho_{o}\right\|_{\mathbf{L}^{\infty}(\mathbb{R} ; \mathbb{R})} e^{\mathcal{L} t}$, as in (2.3) .

Remark 1. The regularity assumptions required in [4] for the functions $v$ and $w$, see [4, Formula (2.2)], are actually less restrictive than (2.2). Indeed, to guarantee the existence of solutions and to obtain the a priori estimates above, it is sufficient that

$$
v \in\left(\mathbf{C}^{2} \cap \mathbf{W}^{1, \infty}\right)(\mathbb{R} ; \mathbb{R}) \quad \text { and } \quad w \in\left(\mathbf{C}^{2} \cap \mathbf{W}^{2, \infty}\right)(\mathbb{R} ; \mathbb{R}) .
$$

Aim of this paper is to study the stability of solutions to (1.1) with respect to both the kernel $w$ and the velocity function $v$. The following Theorem states the $\mathbf{L}^{\mathbf{1}}$-Lipschitz continuous dependence of solutions to (1.1) on both the initial datum and the kernel function.

Theorem 1. Let $T>0$. Fix $f$ and $v$ satisfying (2.1) and (2.2) respectively. Fix $\rho_{o}, \tilde{\rho}_{o} \in$ $\mathbf{L}^{\infty}(\mathbb{R} ; \mathbb{R})$. Let $w, \tilde{w} \in\left(\mathbf{C}^{2} \cap \mathbf{W}^{1,1} \cap \mathbf{W}^{2, \infty}\right)(\mathbb{R} ; \mathbb{R})$. Call $\rho$ and $\tilde{\rho}$ the solutions, in the sense of Definition 1, to the following problems respectively

$$
\begin{aligned}
& \left\{\begin{array}{l}
\partial_{t} \rho+\partial_{x}(f(t, x, \rho) V(t, x))=0 \\
\rho(0, x)=\rho_{o}(x)
\end{array} \quad \text { where } \quad V(t, x)=v((\rho(t) * w)(x)),\right. \\
& \left\{\begin{array}{l}
\partial_{t} \tilde{\rho}+\partial_{x}(f(t, x, \tilde{\rho}) \tilde{V}(t, x))=0 \\
\tilde{\rho}(0, x)=\tilde{\rho}_{o}(x)
\end{array} \quad \text { where } \quad \tilde{V}(t, x)=v((\tilde{\rho}(t) * \tilde{w})(x)) .\right.
\end{aligned}
$$


Then, for any $t \in[0, T]$, the following estimate holds

$$
\|\rho(t)-\tilde{\rho}(t)\|_{\mathbf{L}^{\mathbf{1}(\mathbb{R} ; \mathbb{R})}} \leq\left(\left\|\rho_{o}-\tilde{\rho}_{o}\right\|_{\mathbf{L}^{\mathbf{1}}(\mathbb{R} ; \mathbb{R})}+a(t)\|w-\tilde{w}\|_{\mathbf{W}^{1,1}(\mathbb{R} ; \mathbb{R})}\right) \exp \left(\int_{0}^{t} b(r) \mathrm{d} r\right),
$$

where $a(t)$ and $b(t)$ depend on various norms of the initial data and of the functions $f, v, w$ and $\tilde{w}$, see (3.54) and (3.55).

The $\mathbf{L}^{\mathbf{1}}$-Lipschitz continuous dependence of solutions to (1.1) on the velocity function $v$ is ensured by the following Theorem.

Theorem 2. Let $T>0$. Fix $f$ and $w$ satisfying (2.1) and (2.2) respectively. Fix $\rho_{o} \in$ $\mathbf{L}^{\infty}(\mathbb{R} ; \mathbb{R})$. Let $v, \tilde{v} \in\left(\mathbf{C}^{2} \cap \mathbf{W}^{2, \infty}\right)(\mathbb{R} ; \mathbb{R})$. Call $\rho$ and $\tilde{\rho}$ the solutions, in the sense of Definition 1 , to the following problems respectively

$$
\begin{aligned}
& \left\{\begin{array}{l}
\partial_{t} \rho+\partial_{x}(f(t, x, \rho) V(t, x))=0 \\
\rho(0, x)=\rho_{o}(x)
\end{array} \quad \text { where } V(t, x)=v((\rho(t) * w)(x)),\right. \\
& \left\{\begin{array}{l}
\partial_{t} \tilde{\rho}+\partial_{x}(f(t, x, \tilde{\rho}) \tilde{V}(t, x))=0 \\
\tilde{\rho}(0, x)=\rho_{o}(x)
\end{array} \quad \text { where } \quad \tilde{V}(t, x)=\tilde{v}((\tilde{\rho}(t) * w)(x)) .\right.
\end{aligned}
$$

Then, for any $t \in[0, T]$, the following estimate holds

$$
\|\rho(t)-\tilde{\rho}(t)\|_{\mathbf{L}^{\mathbf{1}(\mathbb{R} ; \mathbb{R})}} \leq\left(c_{1}(t)\|v-\tilde{v}\|_{\mathbf{L}^{\infty}(\mathbb{R} ; \mathbb{R})}+c_{2}(t)\left\|v^{\prime}-\tilde{v}^{\prime}\right\|_{\mathbf{L}^{\infty}(\mathbb{R} ; \mathbb{R})}\right) \exp \left(\int_{0}^{t} c_{3}(s) \mathrm{d} s\right),
$$

where the $c_{i}(t), i=1,2,3$, depend on various norms of the initial data and of the functions $f, v, \tilde{v}$ and $w$, see (3.60), (3.61) and (3.62).

\section{Proofs}

The Lemma below is the building block of both Theorem 1 and Theorem 2 ,

Lemma 4. Let $T>0$. Fix $f$ satisfying (2.1) and $V, \tilde{V} \in\left(\mathbf{C}^{2} \cap \mathbf{W}^{2, \infty}\right)(\mathbb{R} \times \mathbb{R} ; \mathbb{R})$. Fix $\rho_{o}, \tilde{\rho}_{o} \in \mathbf{L}^{\infty}(\mathbb{R} ; \mathbb{R})$. Call $\rho$ and $\tilde{\rho}$ the solutions to the following problems

$$
\left\{\begin{array} { l } 
{ \partial _ { t } \rho + \partial _ { x } ( f ( t , x , \rho ) V ( t , x ) ) = 0 } \\
{ \rho ( 0 , x ) = \rho _ { o } ( x ) }
\end{array} \quad \text { and } \quad \left\{\begin{array}{l}
\partial_{t} \tilde{\rho}+\partial_{x}(f(t, x, \tilde{\rho}) \tilde{V}(t, x))=0 \\
\tilde{\rho}(0, x)=\tilde{\rho}_{o}(x)
\end{array}\right.\right.
$$

Then, for any $\tau, t \in] 0, T[$, with $\tau<t$, the following estimate holds

$$
\begin{gathered}
\int_{\mathbb{R}}|\rho(\tau, x)-\tilde{\rho}(\tau, x)| \mathrm{d} x-\int_{\mathbb{R}}|\rho(t, x)-\tilde{\rho}(t, x)| \mathrm{d} x \\
+\int_{\tau}^{t} \int_{\mathbb{R}}\left\{\left|\partial_{x} \tilde{V}(s, x)-\partial_{x} V(s, x)\right||f(s, x, \rho(s, x))|\right. \\
+|\tilde{V}(s, x)-V(s, x)|\left|\partial_{x} f(s, x, \rho(s, x))\right| \\
\left.+|\tilde{V}(s, x)-V(s, x)|\left|\partial_{\rho} f(s, x, \rho(s, x))\right|\left|\partial_{x} \rho(s, x)\right|\right\} \mathrm{d} x \mathrm{~d} s \geq 0 .
\end{gathered}
$$


Proof. The proof is based on the doubling of variables method introduced by Kružkov in [18]. In particular, we follow the lines of [16, Theorem 1.3], although there the flux function has the form $l(x) g(\rho)$, while here it is of the form $f(t, x, \rho) V(t, x)$. The dependence on time does not add any difficulties in the proof, while the dependence of $f$ on the space variable $x$ produces additional terms.

Let $\varphi \in \mathbf{C}_{c}^{\infty}(] 0 ; T\left[\times \mathbb{R} ; \mathbb{R}^{+}\right)$be a test function as in the definition of solution by Kružkov. Let $Y \in \mathbf{C}_{c}^{\infty}\left(\mathbb{R} ; \mathbb{R}^{+}\right)$be such that

$$
Y(z)=Y(-z), \quad Y(z)=0 \text { for }|z| \geq 1, \quad \int_{\mathbb{R}} Y(z) \mathrm{d} z=1,
$$

and define $Y_{h}=\frac{1}{h} Y\left(\frac{z}{h}\right)$. Obviously $Y_{h} \in \mathbf{C}_{c}^{\infty}\left(\mathbb{R} ; \mathbb{R}^{+}\right), Y_{h}(-z)=Y_{h}(z), Y_{h}(z)=0$ for $|z| \geq h$, $\int_{\mathbb{R}} Y_{h}(z) \mathrm{d} z=1$ and $Y_{h} \rightarrow \delta_{0}$ as $h \rightarrow 0$, where $\delta_{0}$ is the Dirac delta in 0. Define, for $h>0$,

$$
\psi_{h}(t, x, s, y)=\varphi\left(\frac{t+s}{2}, \frac{x+y}{2}\right) Y_{h}(t-s) Y_{h}(x-y)=\varphi(\cdots) Y_{h}(t-s) Y_{h}(x-y) .
$$

Introduce the space $\left.\Pi_{T}=\right] 0, T[\times \mathbb{R}$. We derive the following entropy inequalities for the solutions $\rho=\rho(t, x)$ and $\tilde{\rho}=\tilde{\rho}(s, y)$ to (3.1):

$$
\begin{aligned}
\iiint \int_{\Pi_{T} \times \Pi_{T}}\left\{|\rho-\tilde{\rho}| \partial_{t} \psi_{h}(t, x, s, y)\right. & +\operatorname{sgn}(\rho-\tilde{\rho}) V(t, x)(f(t, x, \rho)-f(t, x, \tilde{\rho})) \partial_{x} \psi_{h}(t, x, s, y) \\
+ & \left.\operatorname{sgn}(\rho-\tilde{\rho}) \partial_{x}[f(t, x, \tilde{\rho}) V(t, x)] \psi_{h}(t, x, s, y)\right\} \mathrm{d} x \mathrm{~d} t \mathrm{~d} y \mathrm{~d} s \geq 0
\end{aligned}
$$

and

$$
\begin{aligned}
\iiint \int_{\Pi_{T} \times \Pi_{T}}\left\{|\tilde{\rho}-\rho| \partial_{t} \psi_{h}(t, x, s, y)\right. & +\operatorname{sgn}(\tilde{\rho}-\rho) \tilde{V}(s, y)(f(s, y, \tilde{\rho})-f(s, y, \rho)) \partial_{y} \psi_{h}(t, x, s, y) \\
+ & \left.\operatorname{sgn}(\tilde{\rho}-\rho) \partial_{y}[f(s, y, \rho) \tilde{V}(s, y)] \psi_{h}(t, x, s, y)\right\} \mathrm{d} x \mathrm{~d} t \mathrm{~d} y \mathrm{~d} s \geq 0 .
\end{aligned}
$$

Summing the two inequalities above and rearranging the terms therein, relying on the explicit form of the function $\psi_{h}$ (3.4), we obtain

$$
\begin{array}{r}
\iiint \int_{\Pi_{T} \times \Pi_{T}}\left\{|\rho(t, x)-\tilde{\rho}(s, y)| \partial_{t} \varphi(\cdots) Y_{h}(t-s) Y_{h}(x-y)\right. \\
+\operatorname{sgn}(\rho-\tilde{\rho})(V(t, x) f(t, x, \rho)-\tilde{V}(s, y) f(s, y, \tilde{\rho})) \partial_{x} \varphi(\cdots) Y_{h}(t-s) Y_{h}(x-y) \\
+\operatorname{sgn}(\rho-\tilde{\rho})(\tilde{V}(s, y) f(s, y, \tilde{\rho})-V(t, x) f(t, x, \tilde{\rho})) \partial_{x} \psi_{h}(t, x, s, y) \\
+\operatorname{sgn}(\rho-\tilde{\rho})(\tilde{V}(s, y) f(s, y, \rho)-V(t, x) f(t, x, \rho)) \partial_{y} \psi_{h}(t, x, s, y) \\
\left.+\operatorname{sgn}(\rho-\tilde{\rho})\left[\partial_{y}(\tilde{V}(s, y) f(s, y, \rho))-\partial_{x}(V(t, x) f(t, x, \tilde{\rho}))\right] \psi_{h}(t, x, s, y)\right\} \\
\mathrm{d} x \mathrm{~d} t \mathrm{~d} y \mathrm{~d} s \geq 0 .
\end{array}
$$


Consider (3.7) and (3.8): explicit the function $\psi_{h}$ to obtain

$$
\begin{aligned}
& {[((\overrightarrow{3.7})]+[(\overline{3.8})]} \\
= & \frac{\operatorname{sgn}(\rho-\tilde{\rho})}{2} \tilde{V}(s, y)(f(s, y, \tilde{\rho})+f(s, y, \rho)) \partial_{x} \varphi(\cdots) Y_{h}(t-s) Y_{h}(x-y) \\
& -\frac{\operatorname{sgn}(\rho-\tilde{\rho})}{2} V(t, x)(f(t, x, \tilde{\rho})+f(t, x, \rho)) \partial_{x} \varphi(\cdots) Y_{h}(t-s) Y_{h}(x-y) \\
& -\operatorname{sgn}(\rho-\tilde{\rho}) \tilde{V}(s, y)(f(s, y, \tilde{\rho})-f(s, y, \rho)) \varphi(\cdots) Y_{h}(t-s) Y_{h}^{\prime}(x-y) \\
& +\operatorname{sgn}(\rho-\tilde{\rho}) V(t, x)(f(t, x, \rho)-f(t, x, \tilde{\rho})) \varphi(\cdots) Y_{h}(t-s) Y_{h}^{\prime}(x-y) .
\end{aligned}
$$

In (3.9) compute

$$
\begin{aligned}
{[(\underline{3.9})]=\operatorname{sgn}(\rho-\tilde{\rho})\left[\partial_{y} \tilde{V}(s, y) f(s, y, \rho)+\tilde{V}(s, y) \partial_{y} f(s, y, \rho)\right.} \\
\left.\quad-\partial_{x} V(t, x) f(t, x, \tilde{\rho})-V(t, x) \partial_{x} f(t, x, \tilde{\rho})\right] \psi_{h}(t, x, s, y) .
\end{aligned}
$$

Introduce the following notation

$$
F(t, x, \rho(t, x), \tilde{\rho}(s, y))=\operatorname{sgn}(\rho(t, x)-\tilde{\rho}(s, y))(f(t, x, \rho(t, x))-f(t, x, \tilde{\rho}(s, y))),
$$

so that (3.12) - (3.13) now reads

$$
\begin{aligned}
& \iiint \int_{\Pi_{T} \times \Pi_{T}}[(\underline{(3.12})]+[(\underline{3.13})] \mathrm{d} x \mathrm{~d} t \mathrm{~d} y \mathrm{~d} s \\
= & \iiint \int_{\Pi_{T} \times \Pi_{T}}(V(t, x) F(t, x, \rho, \tilde{\rho})-\tilde{V}(s, y) F(s, y, \rho, \tilde{\rho})) \varphi(\cdots) Y_{h}(t-s) Y_{h}^{\prime}(x-y) \mathrm{d} x \mathrm{~d} t \mathrm{~d} y \mathrm{~d} s \\
= & -\iiint \int_{\Pi_{T} \times \Pi_{T}}\left(V(t, x) \frac{\mathrm{d}}{\mathrm{d} x} F(t, x, \rho, \tilde{\rho})-\tilde{V}(s, y) \frac{\mathrm{d}}{\mathrm{d} x} F(s, y, \rho, \tilde{\rho})\right) \psi_{h}(t, x, s, y) \mathrm{d} x \mathrm{~d} t \mathrm{~d} y \mathrm{~d} s \\
& -\iiint_{\Pi_{T} \times \Pi_{T}} \partial_{x} V(t, x) F(t, x, \rho, \tilde{\rho}) \psi_{h}(t, x, s, y) \mathrm{d} x \mathrm{~d} t \mathrm{~d} y \mathrm{~d} s \\
& -\iiint \int_{\Pi_{T} \times \Pi_{T}} \frac{1}{2}(V(t, x) F(t, x, \rho, \tilde{\rho})-\tilde{V}(s, y) F(s, y, \rho, \tilde{\rho})) \partial_{x} \varphi(\cdots) \\
& \times Y_{h}(t-s) Y_{h}(x-y) \mathrm{d} x \mathrm{~d} t \mathrm{~d} y \mathrm{~d} s
\end{aligned}
$$

where we also integrate by parts. Combine the integrand of (3.17) together with (3.14) to get

$$
\begin{aligned}
& -\partial_{x} V(t, x) F(t, x, \rho, \tilde{\rho}) \psi_{h}(t, x, s, y)+[[(\underline{3.14})] \\
= & \operatorname{sgn}(\rho-\tilde{\rho})\left(\partial_{y} \tilde{V}(s, y) f(s, y, \rho)-\partial_{x} V(t, x) f(t, x, \rho)\right) \psi_{h}(t, x, s, y) \\
& +\operatorname{sgn}(\rho-\tilde{\rho})\left(\tilde{V}(s, y) \partial_{y} f(s, y, \rho)-V(t, x) \partial_{x} f(t, x, \tilde{\rho})\right) \psi_{h}(t, x, s, y) .
\end{aligned}
$$

Observe that the following equality holds

$$
\iiint_{\Pi_{T} \times \Pi_{T}}[(\sqrt{3.6})]+[(\underline{3.10})]+[(\underline{3.11})]+[(\underline{3.18})] \mathrm{d} x \mathrm{~d} t \mathrm{~d} y \mathrm{~d} s
$$




$$
=\iiint_{\Pi_{T} \times \Pi_{T}} \operatorname{sgn}(\rho-\tilde{\rho}) \tilde{V}(s, y)(f(s, y, \rho)-f(s, y, \tilde{\rho})) \partial_{x} \varphi(\cdots) Y_{h}(t-s) Y_{h}(x-y) \mathrm{d} x \mathrm{~d} t \mathrm{~d} y \mathrm{~d} s .
$$

We are therefore left with

$$
\iiint_{\Pi_{T} \times \Pi_{T}}[(\underline{(3.5)})]+[(\underline{3.16})]+[(\underline{3.19})]+[(\underline{3.20})]+[(\underline{(3.21)}] \mathrm{d} x \mathrm{~d} t \mathrm{~d} y \mathrm{~d} s \geq 0 .
$$

Let now $h$ go to 0 . The terms in (3.5) and (3.21) can be treated exactly as in [18, leading to

$$
\begin{aligned}
& \lim _{h \rightarrow 0+} \iiint_{\Pi_{T} \times \Pi_{T}}\{[(\underline{(3.5)})]+[(\underline{3.21})]\} \mathrm{d} x \mathrm{~d} t \mathrm{~d} y \mathrm{~d} s \\
= & \iint_{\Pi_{T}}\left\{|\rho(t, x)-\tilde{\rho}(t, x)| \partial_{t} \varphi(t, x)\right. \\
& \left.+\operatorname{sgn}(\rho(t, x)-\tilde{\rho}(t, x)) \tilde{V}(t, x)(f(t, x, \rho(t, x))-f(t, x, \tilde{\rho}(t, x))) \partial_{x} \varphi(t, x)\right\} \mathrm{d} x \mathrm{~d} t .
\end{aligned}
$$

Regarding (3.19), we simplify the notation by introducing the map

$$
\Upsilon(t, x, s, y)=\operatorname{sgn}(\rho(t, x)-\tilde{\rho}(s, y))\left(\partial_{y} \tilde{V}(s, y) f(s, y, \rho)-\partial_{x} V(t, x) f(t, x, \rho)\right) \varphi\left(\frac{t+s}{2}, \frac{x+y}{2}\right),
$$

so that

\section{$[(3.19)]$}

$$
\begin{aligned}
= & \Upsilon(t, x, s, y) Y_{h}(t-s) Y_{h}(x-y) \\
= & \Upsilon(t, x, t, x) Y_{h}(t-s) Y_{h}(x-y)+(\Upsilon(t, x, s, y)-\Upsilon(t, x, t, x)) Y_{h}(t-s) Y_{h}(x-y) \\
= & \operatorname{sgn}(\rho(t, x)-\tilde{\rho}(t, x))\left(\partial_{x} \tilde{V}(t, x)-\partial_{x} V(t, x)\right) f(t, x, \rho) \varphi(t, x) Y_{h}(t-s) Y_{h}(x-y) \\
& +(\Upsilon(t, x, s, y)-\Upsilon(t, x, t, x)) Y_{h}(t-s) Y_{h}(x-y) .
\end{aligned}
$$

It is immediate to see that

$$
\begin{aligned}
& \iiint_{\Pi_{T} \times \Pi_{T}}[(\sqrt{3.25})] \mathrm{d} x \mathrm{~d} t \mathrm{~d} y \mathrm{~d} s \\
= & \iint_{\Pi_{T}} \operatorname{sgn}(\rho(t, x)-\tilde{\rho}(t, x))\left(\partial_{x} \tilde{V}(t, x)-\partial_{x} V(t, x)\right) f(t, x, \rho(t, x)) \varphi(t, x) \mathrm{d} x \mathrm{~d} t .
\end{aligned}
$$

Concerning (3.26), it vanishes as $h$ goes to 0 when integrated over $\Pi_{T} \times \Pi_{T}$. Indeed, recall that $\left|Y_{h}\right| \leq(Y(0) / h) \chi_{[-h, h]}$ and apply [12, Lemma 6.2], see also [18, Lemma 2], with $N=3$, $X=(x, t, x), Y=(x, t, y)$ and

$$
w(s, Y)=\frac{(Y(0))^{2}}{h^{2}} \Upsilon(t, x, s, y) .
$$


Focus the attention on (3.16). With abuse of notation, since the function $F$ is only Lipschitz continuous with respect to $\rho$, we write

$$
\begin{aligned}
& \frac{\mathrm{d}}{\mathrm{d} x} F(t, x, \rho(t, x), \tilde{\rho}(s, y)) \\
= & \partial_{x} F(t, x, \rho(t, x), \tilde{\rho}(s, y))+\partial_{\rho} F(t, x, \rho(t, x), \tilde{\rho}(s, y)) \partial_{x} \rho(t, x) \\
= & \operatorname{sgn}(\rho(t, x)-\tilde{\rho}(s, y))\left(\partial_{x} f(t, x, \rho(t, x))-\partial_{x} f(t, x, \tilde{\rho}(s, y))\right) \\
& +\partial_{\rho} F(t, x, \rho(t, x), \tilde{\rho}(s, y)) \partial_{x} \rho(t, x)
\end{aligned}
$$

and

$$
\begin{aligned}
& \frac{\mathrm{d}}{\mathrm{d} x} F(s, y, \rho(t, x), \tilde{\rho}(s, y)) \\
= & \partial_{\rho} F(s, y, \rho(t, x), \tilde{\rho}(s, y)) \partial_{x} \rho(t, x) \\
= & \partial_{\rho} F(t, x, \rho(t, x), \tilde{\rho}(t, x)) \partial_{x} \rho(t, x) \\
& +\left(\partial_{\rho} F(s, y, \rho(t, x), \tilde{\rho}(s, y))-\partial_{\rho} F(t, x, \rho(t, x), \tilde{\rho}(t, x))\right) \partial_{x} \rho(t, x) .
\end{aligned}
$$

In particular observe that we can combine (3.20) with (3.28) to get

$$
\begin{aligned}
& {[(\underline{3.20})]-V(t, x) \operatorname{sgn}(\rho(t, x)-\tilde{\rho}(s, y))\left(\partial_{x} f(t, x, \rho(t, x))-\partial_{x} f(t, x, \tilde{\rho}(s, y))\right) \psi_{h}(t, x, s, y)} \\
& =\operatorname{sgn}(\rho(t, x)-\tilde{\rho}(s, y))\left(\tilde{V}(s, y) \partial_{y} f(s, y, \rho)-V(t, x) \partial_{x} f(t, x, \rho)\right) \psi_{h}(t, x, s, y) \text {. }
\end{aligned}
$$

An application of [12, Lemma 6.2] yields

$$
\begin{aligned}
& \lim _{h \rightarrow 0} \iiint_{\Pi_{T} \times \Pi_{T}}[(\sqrt{3.32})] \mathrm{d} x \mathrm{~d} t \mathrm{~d} y \mathrm{~d} s \\
= & \iint_{\Pi_{T}} \operatorname{sgn}(\rho(t, x)-\tilde{\rho}(t, x))(\tilde{V}(t, x)-V(t, x)) \partial_{x} f(t, x, \rho(t, x)) \varphi(t, x) \mathrm{d} x \mathrm{~d} t .
\end{aligned}
$$

In order to deal with the remaining terms, i.e. (3.29), (3.30) and (3.31), we need to introduce a regularisation of the sign function. In particular, for $\alpha>0$ set

$$
s_{\alpha}(u)=\left(\operatorname{sgn} * Y_{\alpha}\right)(u) .
$$

Observe that $s_{\alpha}^{\prime}(u)=\frac{2}{\alpha} Y\left(\frac{u}{\alpha}\right)$. Recall the definition of the map $F(\underline{3.15})$ and compute

$$
\begin{gathered}
\iiint \int_{\Pi_{T} \times \Pi_{T}}-V(t, x) \times[(\underline{3.29})] \times \psi_{h}(t, x, s, y) \mathrm{d} x \mathrm{~d} t \mathrm{~d} y \mathrm{~d} s \\
=\lim _{\alpha \rightarrow 0} \iiint \int_{\Pi_{T} \times \Pi_{T}}\left\{s_{\alpha}^{\prime}(\rho(t, x)-\tilde{\rho}(s, y))(f(t, x, \rho(t, x))-f(t, x, \tilde{\rho}(s, y)))\right. \\
\left.\quad+s_{\alpha}(\rho(t, x)-\tilde{\rho}(s, y)) \partial_{\rho} f(t, x, \rho(t, x))\right\} \\
\quad \times(-V(t, x)) \partial_{x} \rho(t, x) \psi_{h}(t, x, s, y) \mathrm{d} x \mathrm{~d} t \mathrm{~d} y \mathrm{~d} s .
\end{gathered}
$$


By the Dominated Convergence Theorem, as $\alpha$ goes to 0 , we get

$$
\iiint_{\Pi_{T} \times \Pi_{T}}[(\sqrt{3.34})] \times[(\sqrt{3.36})] \mathrm{d} x \mathrm{~d} t \mathrm{~d} y \mathrm{~d} s \rightarrow 0 .
$$

Indeed,

$$
\begin{aligned}
& \left|\frac{2}{\alpha} Y\left(\frac{\rho-\tilde{\rho}}{\alpha}\right)(f(t, x, \rho(t, x))-f(t, x, \tilde{\rho}(s, y))) V(t, x) \partial_{x} \rho(t, x) \psi_{h}(t, x, s, y)\right| \\
\leq & \frac{2}{\alpha} Y\left(\frac{\rho-\tilde{\rho}}{\alpha}\right) \int_{\tilde{\rho}}^{\rho}\left|\partial_{\rho} f(s, y, r)\right| \mathrm{d} r\|V\|_{\mathbf{L}^{\infty}\left(\Pi_{T} ; \mathbb{R}\right)}\left|\partial_{x} \rho(t, x)\right| \psi_{h}(t, x, s, y) \\
\leq & 2\|Y\|_{\mathbf{L}^{\infty}(\mathbb{R} ; \mathbb{R})}\left\|\partial_{\rho} f\right\|_{\mathbf{L}^{\infty}\left(\Pi_{T} \times \mathbb{R} ; \mathbb{R}\right)}\|V\|_{\mathbf{L}^{\infty}\left(\Pi_{T} ; \mathbb{R}\right)}\left|\partial_{x} \rho(t, x)\right| \psi_{h}(t, x, s, y) \quad \in \mathbf{L}^{\mathbf{1}}\left(\Pi_{T} \times \Pi_{T} ; \mathbb{R}\right) .
\end{aligned}
$$

Therefore we have

$$
\begin{aligned}
& \iiint \int_{\Pi_{T} \times \Pi_{T}}-V(t, x) \times[(\sqrt{3.29})] \times \psi_{h}(t, x, s, y) \mathrm{d} x \mathrm{~d} t \mathrm{~d} y \mathrm{~d} s \\
= & -\iiint \int_{\Pi_{T} \times \Pi_{T}} \operatorname{sgn}(\rho(t, x)-\tilde{\rho}(s, y)) V(t, x) \partial_{\rho} f(t, x, \rho(t, x)) \partial_{x} \rho(t, x) \psi_{h}(t, x, s, y) \mathrm{d} x \mathrm{~d} t \mathrm{~d} y \mathrm{~d} s .
\end{aligned}
$$

The term

$$
\iiint_{\Pi_{T} \times \Pi_{T}} \tilde{V}(s, y) \times[(\underline{(3.30})] \times \psi_{h}(t, x, s, y) \mathrm{d} x \mathrm{~d} t \mathrm{~d} y \mathrm{~d} s
$$

can be treated exactly in the same way, leading to

$$
\iiint_{\Pi_{T} \times \Pi_{T}} \operatorname{sgn}(\rho(t, x)-\tilde{\rho}(t, x)) \tilde{V}(s, y) \partial_{\rho} f(t, x, \rho(t, x)) \partial_{x} \rho(t, x) \psi_{h}(t, x, s, y) \mathrm{d} x \mathrm{~d} t \mathrm{~d} y \mathrm{~d} s .
$$

Introduce now the notation

$$
\Upsilon(s, y)=\operatorname{sgn}(\rho(t, x)-\tilde{\rho}(t, x)) \tilde{V}(s, y)-\operatorname{sgn}(\rho(t, x)-\tilde{\rho}(s, y)) V(t, x)
$$

and apply [12, Lemma 6.2]:

$$
\begin{aligned}
& \lim _{h \rightarrow 0}[(\underline{\underline{3.38}})]+[(\underline{3.37})] \\
= & \iint_{\Pi_{T}} \operatorname{sgn}(\rho(t, x)-\tilde{\rho}(t, x))(\tilde{V}(t, x)-V(t, x)) \partial_{\rho} f(t, x, \rho(t, x)) \partial_{x} \rho(t, x) \varphi(t, x) d x \mathrm{~d} t .
\end{aligned}
$$

In order to deal with the last term, i.e. (3.31), exploit the same regularisation of the sign function as above and compute

$$
\iiint_{\Pi_{T} \times \Pi_{T}} \tilde{V}(s, y) \times[(\underline{3.31})] \times \psi_{h}(t, x, s, y) \mathrm{d} x \mathrm{~d} t \mathrm{~d} y \mathrm{~d} s
$$




$$
\begin{aligned}
=\lim _{\alpha \rightarrow 0} \iiint_{\Pi_{T} \times \Pi_{T}} & {\left[s_{\alpha}^{\prime}(\rho(t, x)-\tilde{\rho}(s, y))(f(s, y, \rho(t, x))-f(s, y, \tilde{\rho}(s, y)))\right.} \\
& -s_{\alpha}^{\prime}(\rho(t, x)-\tilde{\rho}(t, x))(f(t, x, \rho(t, x))-f(t, x, \tilde{\rho}(t, x))) \\
& +s_{\alpha}(\rho(t, x)-\tilde{\rho}(s, y)) \partial_{\rho} f(s, y, \rho(t, x)) \\
& \left.-s_{\alpha}(\rho(t, x)-\tilde{\rho}(t, x)) \partial_{\rho} f(t, x, \rho(t, x))\right] \\
\times & \tilde{V}(s, y) \partial_{x} \rho(t, x) \psi_{h}(t, x, s, y) \mathrm{d} x \mathrm{~d} t \mathrm{~d} y \mathrm{~d} s .
\end{aligned}
$$

By the Dominated Convergence Theorem, as $\alpha$ goes to 0 , we get

$$
\iiint_{\Pi_{T} \times \Pi_{T}}[(\underline{3.41})] \times[(\underline{3.43})] \mathrm{d} x \mathrm{~d} t \mathrm{~d} y \mathrm{~d} s \rightarrow 0, \quad \iiint \int_{\Pi_{T} \times \Pi_{T}}[(\underline{3.42})] \times[(\underline{3.43})] \mathrm{d} x \mathrm{~d} t \mathrm{~d} y \mathrm{~d} s \rightarrow 0 .
$$

Indeed,

$$
\begin{aligned}
& \left|\frac{2}{\alpha} Y\left(\frac{\rho-\tilde{\rho}}{\alpha}\right)(f(s, y, \rho(t, x))-f(s, y, \tilde{\rho}(s, y))) \tilde{V}(s, y) \partial_{x} \rho(t, x) \psi_{h}(t, x, s, y)\right| \\
\leq & \frac{2}{\alpha} Y\left(\frac{\rho-\tilde{\rho}}{\alpha}\right) \int_{\tilde{\rho}}^{\rho}\left|\partial_{\rho} f(s, y, r)\right| \mathrm{d} r\|\tilde{V}\|_{\mathbf{L}^{\infty}\left(\Pi_{T} ; \mathbb{R}\right)}\left|\partial_{x} \rho(t, x)\right| \psi_{h}(t, x, s, y) \\
\leq & 2\|Y\|_{\mathbf{L}^{\infty}(\mathbb{R} ; \mathbb{R})}\left\|\partial_{\rho} f\right\|_{\mathbf{L}^{\infty}\left(\Pi_{T} \times \mathbb{R} ; \mathbb{R}\right)}\|\tilde{V}\|_{\mathbf{L}^{\infty}\left(\Pi_{T} ; \mathbb{R}\right)}\left|\partial_{x} \rho(t, x)\right| \psi_{h}(t, x, s, y) \quad \in \mathbf{L}^{\mathbf{1}\left(\Pi_{T} \times \Pi_{T} ; \mathbb{R}\right) .}
\end{aligned}
$$

Therefore we have

$$
\begin{aligned}
& \left.\left.=\iint_{\Pi_{T} \times \Pi_{T}}^{[(\overline{3.40})}\right]\right] \\
& \quad \times \tilde{V}(s, y) \partial_{x} \rho(t, x) \psi_{h}(t, x, s, y) \mathrm{d} x \mathrm{~d} t \mathrm{~d} y \mathrm{~d} s .
\end{aligned}
$$

Introduce the notation $\Upsilon(s, y)=\operatorname{sgn}(\rho(t, x)-\tilde{\rho}(s, y)) \partial_{\rho} f(s, y, \rho(t, x))$ and rewrite the equality above as follows

$$
[\underline{(3.40)})] \leq\|\tilde{V}\|_{\mathbf{L}^{\infty}\left(\Pi_{T} ; \mathbb{R}\right)} \iiint_{\Pi_{T} \times \Pi_{T}}|\Upsilon(s, y)-\Upsilon(t, x)|\left|\partial_{x} \rho(t, x)\right| \psi_{h}(t, x, s, y) \mathrm{d} x \mathrm{~d} t \mathrm{~d} y \mathrm{~d} s,
$$

the left hand side clearly vanishing as $h$ goes to 0 , thanks to [12, Lemma 6.2] and to the fact that $\rho$ has bounded variation.

Collecting together all the estimates obtained in (3.23), (3.24), (3.27), (3.33) and (3.39), we get

$$
\begin{aligned}
& \lim _{h \rightarrow 0}[(\overline{3.22})] \\
= & \iint_{\Pi_{T}}\left\{|\rho(t, x)-\tilde{\rho}(t, x)| \partial_{t} \varphi(t, x)\right. \\
& +\operatorname{sgn}(\rho(t, x)-\tilde{\rho}(t, x)) \tilde{V}(t, x)(f(t, x, \rho(t, x))-f(t, x, \tilde{\rho}(t, x))) \partial_{x} \varphi(t, x)
\end{aligned}
$$




$$
\begin{aligned}
& +\operatorname{sgn}(\rho(t, x)-\tilde{\rho}(t, x))\left(\partial_{x} \tilde{V}(t, x)-\partial_{x} V(t, x)\right) f(t, x, \rho) \varphi(t, x) \\
& +\operatorname{sgn}(\rho(t, x)-\tilde{\rho}(t, x))(\tilde{V}(t, x)-V(t, x)) \partial_{x} f(t, x, \rho(t, x)) \varphi(t, x) \\
& \left.+\operatorname{sgn}(\rho(t, x)-\tilde{\rho}(t, x))(\tilde{V}(t, x)-V(t, x)) \partial_{\rho} f(t, x, \rho(t, x)) \partial_{x} \rho(t, x) \varphi(t, x)\right\} \mathrm{d} x \mathrm{~d} t .
\end{aligned}
$$

Let now $h>0$ and $r>1$. Fix $0<\tau<t<T$, define

$$
\Phi_{h}(s)=\alpha_{h}(s-\tau)-\alpha_{h}(s-t), \quad \text { where } \quad \alpha_{h}(z)=\int_{-\infty}^{z} Y_{h}(\zeta) \mathrm{d} \zeta
$$

and

$$
\Psi_{r}(x)=\int_{\mathbb{R}} Y(|x-y|) \chi_{\{|y|<r\}}(y) \mathrm{d} y .
$$

Observe that, as $h$ goes to $0, \Phi_{h} \rightarrow \chi_{[\tau, t]}$, and $\Phi_{h}^{\prime} \rightarrow \delta_{\tau}-\delta_{t}$. Moreover, $\Psi_{r}^{\prime}(x)=0$ for $|x|<r-1$ or $|x|>r+1$ and, as $r$ tends to $+\infty, \Psi_{r} \rightarrow \chi_{\mathbb{R}}$. Choose $\varphi(t, x)=\Phi_{h}(t) \Psi_{r}(x)$ in $[(3.44) \cdots(3.45)]$ and pass to the limits $h \rightarrow 0$ and $r \rightarrow+\infty$ to obtain the desired estimate $[(\underline{3.2})-(3.3)]$ :

$$
\begin{gathered}
\int_{\mathbb{R}}|\rho(\tau, x)-\tilde{\rho}(\tau, x)| \mathrm{d} x-\int_{\mathbb{R}}|\rho(t, x)-\tilde{\rho}(t, x)| \mathrm{d} x \\
+\int_{\tau}^{t} \int_{\mathbb{R}}\left\{\left|\partial_{x} \tilde{V}(s, x)-\partial_{x} V(s, x)\right||f(s, x, \rho(s, x))|\right. \\
\quad+|\tilde{V}(s, x)-V(s, x)|\left|\partial_{x} f(s, x, \rho(s, x))\right| \\
\left.+|\tilde{V}(s, x)-V(s, x)|\left|\partial_{\rho} f(s, x, \rho(s, x))\right|\left|\partial_{x} \rho(s, x)\right|\right\} \mathrm{d} x \mathrm{~d} s \geq 0 .
\end{gathered}
$$

Proof of Theorem 1, We can apply Lemma 4 to problems (2.6) and (2.7). By Lemma 3 , with obvious notation, for all $t \in[0, T]$ we have

$$
\|\rho(t)\|_{\mathbf{L}^{\infty}(\mathbb{R} ; \mathbb{R})} \leq\left\|\rho_{o}\right\|_{\mathbf{L}^{\infty}(\mathbb{R} ; \mathbb{R})} e^{\mathcal{L} t}=M_{t}, \quad\|\tilde{\rho}(t)\|_{\mathbf{L}^{\infty}(\mathbb{R} ; \mathbb{R})} \leq\left\|\tilde{\rho}_{o}\right\|_{\mathbf{L}^{\infty}(\mathbb{R} ; \mathbb{R})} e^{\tilde{\mathcal{L}} t}=\tilde{M}_{t} .
$$

For the sake of simplicity introduce the space

$$
\Sigma_{t}=[0, t] \times \mathbb{R} \times\left[0, \max \left\{M_{t}, \tilde{M}_{t}\right\}\right] .
$$

Let $\tau \rightarrow 0$ in $[(\underline{3.2}) \cdots(\underline{3.3})]$ :

$$
\begin{aligned}
\|\rho(t)-\tilde{\rho}(t)\|_{\mathbf{L}^{1}(\mathbb{R} ; \mathbb{R})} \leq & \left\|\rho_{o}-\tilde{\rho}_{o}\right\|_{\mathbf{L}^{1}(\mathbb{R} ; \mathbb{R})} \\
& +\int_{0}^{t}\|f\|_{\mathbf{L}^{\infty}\left(\Sigma_{s} ; \mathbb{R}\right)} \int_{\mathbb{R}}\left|\partial_{x} V(s, x)-\partial_{x} \tilde{V}(s, x)\right| \mathrm{d} x \mathrm{~d} s \\
& +\int_{0}^{t}\left\|\partial_{x} f\right\|_{\mathbf{L}^{\infty}\left(\Sigma_{s} ; \mathbb{R}\right)} \int_{\mathbb{R}}|\tilde{V}(s, x)-V(s, x)| \mathrm{d} x \mathrm{~d} s \\
& +\int_{0}^{t}\left\|\partial_{\rho} f\right\|_{\mathbf{L}^{\infty}\left(\Sigma_{s} ; \mathbb{R}\right)} \int_{\mathbb{R}}\left|\partial_{x} \rho(s, x)\right||V(s, x)-\tilde{V}(s, x)| \mathrm{d} x \mathrm{~d} s .
\end{aligned}
$$


Consider (3.48). By the definitions of $V$ and $\tilde{V}$, compute

$$
\begin{aligned}
& \int_{\mathbb{R}}\left|\partial_{x} V(s, x)-\partial_{x} \tilde{V}(s, x)\right| \mathrm{d} x \\
& =\int_{\mathbb{R}}\left|v^{\prime}((\rho(s) * w)(x))\left(\rho(s) * \partial_{x} w\right)(x)-v^{\prime}((\tilde{\rho}(s) * \tilde{w})(x))\left(\tilde{\rho}(s) * \partial_{x} \tilde{w}\right)(x)\right| \mathrm{d} x \\
& \leq\left\|v^{\prime}\right\|_{\mathbf{L}^{\infty}(\mathbb{R} ; \mathbb{R})}\left(\|\rho(s)-\tilde{\rho}(s)\|_{\mathbf{L}^{1}(\mathbb{R} ; \mathbb{R})} \min \left\{\left\|\partial_{x} w\right\|_{\mathbf{L}^{\mathbf{1}}(\mathbb{R} ; \mathbb{R})},\left\|\partial_{x} \tilde{w}\right\|_{\mathbf{L}^{1}(\mathbb{R} ; \mathbb{R})}\right\}\right. \\
& \left.+\left\|\partial_{x} w-\partial_{x} \tilde{w}\right\|_{\mathbf{L}^{\mathbf{1}}(\mathbb{R} ; \mathbb{R})} \min \left\{\|\rho(s)\|_{\mathbf{L}^{\mathbf{1}}(\mathbb{R} ; \mathbb{R})},\|\tilde{\rho}(s)\|_{\mathbf{L}^{\mathbf{1}}(\mathbb{R} ; \mathbb{R})}\right\}\right) \\
& +\left\|v^{\prime \prime}\right\|_{\mathbf{L}^{\infty}(\mathbb{R} ; \mathbb{R})} \min \left\{\|\rho(s)\|_{\mathbf{L}^{\mathbf{1}(\mathbb{R} ; \mathbb{R})}}\left\|\partial_{x} w\right\|_{\mathbf{L}^{\infty}(\mathbb{R} ; \mathbb{R})},\|\tilde{\rho}(s)\|_{\mathbf{L}^{1}(\mathbb{R} ; \mathbb{R})}\left\|\partial_{x} \tilde{w}\right\|_{\mathbf{L}^{\infty}(\mathbb{R} ; \mathbb{R})}\right\} \\
& \times\left(\|\rho(s)-\tilde{\rho}(s)\|_{\mathbf{L}^{\mathbf{1}}(\mathbb{R} ; \mathbb{R})} \min \left\{\|w\|_{\mathbf{L}^{\mathbf{1}}(\mathbb{R} ; \mathbb{R})},\|\tilde{w}\|_{\mathbf{L}^{\mathbf{1}}(\mathbb{R} ; \mathbb{R})}\right\}\right. \\
& \left.+\|w-\tilde{w}\|_{\mathbf{L}^{1}(\mathbb{R} ; \mathbb{R})} \min \left\{\|\rho(s)\|_{\mathbf{L}^{1}(\mathbb{R} ; \mathbb{R})},\|\tilde{\rho}(s)\|_{\mathbf{L}^{1}(\mathbb{R} ; \mathbb{R})}\right\}\right) \\
& \leq\left(\left\|v^{\prime}\right\|_{\mathbf{L}^{\infty}(\mathbb{R} ; \mathbb{R})}+\left\|v^{\prime \prime}\right\|_{\mathbf{L}^{\infty}(\mathbb{R} ; \mathbb{R})} \min \left\{\left\|\rho_{o}\right\|_{\mathbf{L}^{1}(\mathbb{R} ; \mathbb{R})}\left\|\partial_{x} w\right\|_{\mathbf{L}^{\infty}(\mathbb{R} ; \mathbb{R})},\left\|\tilde{\rho}_{o}\right\|_{\mathbf{L}^{1}(\mathbb{R} ; \mathbb{R})}\left\|\partial_{x} \tilde{w}\right\|_{\mathbf{L}^{\infty}(\mathbb{R} ; \mathbb{R})}\right\}\right) \\
& \times\left(\|\rho(s)-\tilde{\rho}(s)\|_{\mathbf{L}^{\mathbf{1}(\mathbb{R} ; \mathbb{R})}} \min \left\{\|w\|_{\mathbf{W}^{1,1}(\mathbb{R} ; \mathbb{R})},\|\tilde{w}\|_{\mathbf{W}^{1,1}(\mathbb{R} ; \mathbb{R})}\right\}\right. \\
& \left.+\|w-\tilde{w}\|_{\mathbf{W}^{1,1}(\mathbb{R} ; \mathbb{R})} \min \left\{\left\|\rho_{o}\right\|_{\mathbf{L}^{1}(\mathbb{R} ; \mathbb{R})},\left\|\tilde{\rho}_{o}\right\|_{\mathbf{L}^{1}(\mathbb{R} ; \mathbb{R})}\right\}\right),
\end{aligned}
$$

where we exploit also Lemma 2, Therefore,

$[(3.48)]$

$$
\begin{aligned}
& \leq\left(\left\|v^{\prime}\right\|_{\mathbf{L}^{\infty}(\mathbb{R} ; \mathbb{R})}+\left\|v^{\prime \prime}\right\|_{\mathbf{L}^{\infty}(\mathbb{R} ; \mathbb{R})} \min \left\{\left\|\rho_{o}\right\|_{\mathbf{L}^{1}(\mathbb{R} ; \mathbb{R})}\left\|\partial_{x} w\right\|_{\mathbf{L}^{\infty}(\mathbb{R} ; \mathbb{R})},\left\|\tilde{\rho}_{o}\right\|_{\mathbf{L}^{1}(\mathbb{R} ; \mathbb{R})}\left\|\partial_{x} \tilde{w}\right\|_{\mathbf{L}^{\infty}(\mathbb{R} ; \mathbb{R})}\right\}\right) \\
& \times\left(\min \left\{\|w\|_{\mathbf{W}^{1,1}(\mathbb{R} ; \mathbb{R})},\|\tilde{w}\|_{\mathbf{W}^{1,1}(\mathbb{R} ; \mathbb{R})}\right\} \int_{0}^{t}\|f\|_{\mathbf{L}^{\infty}\left(\Sigma_{s} ; \mathbb{R}\right)}\|\rho(s)-\tilde{\rho}(s)\|_{\mathbf{L}^{1}(\mathbb{R} ; \mathbb{R})} \mathrm{d} s\right. \\
& \left.\quad+\|w-\tilde{w}\|_{\mathbf{W}^{1,1}(\mathbb{R} ; \mathbb{R})}\|f\|_{\mathbf{L}^{\infty}\left(\Sigma_{t} ; \mathbb{R}\right)} \min \left\{\left\|\rho_{o}\right\|_{\mathbf{L}^{1}(\mathbb{R} ; \mathbb{R})},\left\|\tilde{\rho}_{o}\right\|_{\mathbf{L}^{1}(\mathbb{R} ; \mathbb{R})}\right\} t\right) .
\end{aligned}
$$

Consider (3.49): compute

$$
\begin{aligned}
& \int_{\mathbb{R}}|V(s, x)-\tilde{V}(s, x)| \mathrm{d} x \leq\left\|v^{\prime}\right\|_{\mathbf{L}^{\infty}(\mathbb{R} ; \mathbb{R})} \min \left\{\|w\|_{\mathbf{L}^{\mathbf{1}}(\mathbb{R} ; \mathbb{R})},\|\tilde{w}\|_{\mathbf{L}^{1}(\mathbb{R} ; \mathbb{R})}\right\}\|\rho(s)-\tilde{\rho}(s)\|_{\mathbf{L}^{1}(\mathbb{R} ; \mathbb{R})}
\end{aligned}
$$

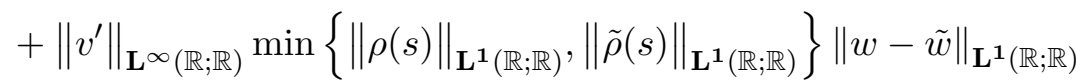

$$
\begin{aligned}
& \leq\left\|v^{\prime}\right\|_{\mathbf{L}^{\infty}(\mathbb{R} ; \mathbb{R})} \min \left\{\|w\|_{\mathbf{L}^{\mathbf{1}(\mathbb{R} ; \mathbb{R})}},\|\tilde{w}\|_{\mathbf{L}^{\mathbf{1}(\mathbb{R} ; \mathbb{R})}}\right\}\|\rho(s)-\tilde{\rho}(s)\|_{\mathbf{L}^{\mathbf{1}(\mathbb{R} ; \mathbb{R})}} \\
& +\left\|v^{\prime}\right\|_{\mathbf{L}^{\infty}(\mathbb{R} ; \mathbb{R})} \min \left\{\left\|\rho_{o}\right\|_{\mathbf{L}^{\mathbf{1}}(\mathbb{R} ; \mathbb{R})},\left\|\tilde{\rho}_{o}\right\|_{\mathbf{L}^{\mathbf{1}}(\mathbb{R} ; \mathbb{R})}\right\}\|w-\tilde{w}\|_{\mathbf{L}^{1}(\mathbb{R} ; \mathbb{R})} .
\end{aligned}
$$

In this way we have

$$
[\underline{(3.49)}] \leq\left\|v^{\prime}\right\|_{\mathbf{L}^{\infty}(\mathbb{R} ; \mathbb{R})} \min \left\{\|w\|_{\mathbf{L}^{1}(\mathbb{R} ; \mathbb{R})},\|\tilde{w}\|_{\mathbf{L}^{1}(\mathbb{R} ; \mathbb{R})}\right\} \int_{0}^{t}\left\|\partial_{x} f\right\|_{\mathbf{L}^{\infty}\left(\Sigma_{s} ; \mathbb{R}\right)}\|\rho(s)-\tilde{\rho}(s)\|_{\mathbf{L}^{1}(\mathbb{R} ; \mathbb{R})} \mathrm{d} s
$$




$$
+\left\|\partial_{x} f\right\|_{\mathbf{L}^{\infty}\left(\Sigma_{t} ; \mathbb{R}\right)}\left\|v^{\prime}\right\|_{\mathbf{L}^{\infty}(\mathbb{R} ; \mathbb{R})} \min \left\{\left\|\rho_{o}\right\|_{\mathbf{L}^{1}(\mathbb{R} ; \mathbb{R})},\left\|\tilde{\rho}_{o}\right\|_{\mathbf{L}^{1}(\mathbb{R} ; \mathbb{R})}\right\}\|w-\tilde{w}\|_{\mathbf{L}^{1}(\mathbb{R} ; \mathbb{R})} t
$$

Finally, consider (3.50) and compute

$$
\begin{aligned}
|V(s, x)-\tilde{V}(s, x)| \leq & \left\|v^{\prime}\right\|_{\mathbf{L}^{\infty}(\mathbb{R} ; \mathbb{R})} \min \left\{\|w\|_{\mathbf{L}^{\infty}(\mathbb{R} ; \mathbb{R})},\|\tilde{w}\|_{\mathbf{L}^{\infty}(\mathbb{R} ; \mathbb{R})}\right\}\|\rho(s)-\tilde{\rho}(s)\|_{\mathbf{L}^{\mathbf{1}(\mathbb{R} ; \mathbb{R})}} \\
& +\left\|v^{\prime}\right\|_{\mathbf{L}^{\infty}(\mathbb{R} ; \mathbb{R})} \min \left\{\|\rho(s)\|_{\mathbf{L}^{\infty}(\mathbb{R} ; \mathbb{R})},\|\tilde{\rho}(s)\|_{\mathbf{L}^{\infty}(\mathbb{R} ; \mathbb{R})}\right\}\|w-\tilde{w}\|_{\mathbf{L}^{\mathbf{1}(\mathbb{R} ; \mathbb{R})}} \\
\leq & \left\|v^{\prime}\right\|_{\mathbf{L}^{\infty}(\mathbb{R} ; \mathbb{R})} \min \left\{\|w\|_{\mathbf{L}^{\infty}(\mathbb{R} ; \mathbb{R})},\|\tilde{w}\|_{\mathbf{L}^{\infty}(\mathbb{R} ; \mathbb{R})}\right\}\|\rho(s)-\tilde{\rho}(s)\|_{\mathbf{L}^{\mathbf{1}(\mathbb{R} ; \mathbb{R})}} \\
& +\left\|v^{\prime}\right\|_{\mathbf{L}^{\infty}(\mathbb{R} ; \mathbb{R})} \min \left\{M_{s}, \tilde{M}_{s}\right\}\|w-\tilde{w}\|_{\mathbf{L}^{\mathbf{1}}(\mathbb{R} ; \mathbb{R})} .
\end{aligned}
$$

Hence,

$$
\begin{aligned}
{[(\underline{3.50})] \leq } & \left\|v^{\prime}\right\|_{\mathbf{L}^{\infty}(\mathbb{R} ; \mathbb{R})} \min \left\{\|w\|_{\mathbf{L}^{\infty}(\mathbb{R} ; \mathbb{R})},\|\tilde{w}\|_{\mathbf{L}^{\infty}(\mathbb{R} ; \mathbb{R})}\right\} \\
& \times \int_{0}^{t}\left\|\partial_{\rho} f\right\|_{\mathbf{L}^{\infty}\left(\Sigma_{s} ; \mathbb{R}\right)} \operatorname{TV}(\rho(s))\|\rho(s)-\tilde{\rho}(s)\|_{\mathbf{L}^{1}(\mathbb{R} ; \mathbb{R})} \mathrm{d} s \\
& +\left\|\partial_{\rho} f\right\|_{\mathbf{L}^{\infty}\left(\Sigma_{t} ; \mathbb{R}\right)} \operatorname{TV}(\rho(t))\left\|v^{\prime}\right\|_{\mathbf{L}^{\infty}(\mathbb{R} ; \mathbb{R})} \min \left\{M_{t}, \tilde{M}_{t}\right\}\|w-\tilde{w}\|_{\mathbf{L}^{\mathbf{1}}(\mathbb{R} ; \mathbb{R})} t .
\end{aligned}
$$

Therefore, the inequality $[(\underline{3.47}) \cdots(\underline{3.50})]$ can be estimated as follows

$$
\begin{aligned}
& \|\rho(t)-\tilde{\rho}(t)\|_{\mathbf{L}^{1}(\mathbb{R} ; \mathbb{R})} \\
\leq & \left\|\rho_{o}-\tilde{\rho}_{o}\right\|_{\mathbf{L}^{1}(\mathbb{R} ; \mathbb{R})}+a(t)\|w-\tilde{w}\|_{\mathbf{W}^{1,1}(\mathbb{R} ; \mathbb{R})}+\int_{0}^{t} b(s)\|\rho(s)-\tilde{\rho}(s)\|_{\mathbf{L}^{1}(\mathbb{R} ; \mathbb{R})} \mathrm{d} s,
\end{aligned}
$$

where, thanks to the total variation estimate provided by Proposition 1,

$$
\begin{aligned}
& a(t) \\
= & t\left[\min \left\{\left\|\rho_{o}\right\|_{\mathbf{L}^{\mathbf{1}(\mathbb{R} ; \mathbb{R})}},\left\|\tilde{\rho}_{o}\right\|_{\mathbf{L}^{\mathbf{1}}(\mathbb{R} ; \mathbb{R})}\right\}\|f\|_{\mathbf{L}^{\infty}\left(\Sigma_{t} ; \mathbb{R}\right)}\right. \\
& \times\left(\left\|v^{\prime}\right\|_{\mathbf{L}^{\infty}(\mathbb{R} ; \mathbb{R})}+\left\|v^{\prime \prime}\right\|_{\mathbf{L}^{\infty}(\mathbb{R} ; \mathbb{R})} \min \left\{\left\|\rho_{o}\right\|_{\mathbf{L}^{\mathbf{1}}(\mathbb{R} ; \mathbb{R})}\left\|\partial_{x} w\right\|_{\mathbf{L}^{\infty}(\mathbb{R} ; \mathbb{R})},\left\|\tilde{\rho}_{o}\right\|_{\mathbf{L}^{\mathbf{1}(\mathbb{R} ; \mathbb{R})}}\left\|\partial_{x} \tilde{w}\right\|_{\mathbf{L}^{\infty}(\mathbb{R} ; \mathbb{R})}\right\}\right) \\
& +\min \left\{\left\|\rho_{o}\right\|_{\mathbf{L}^{\mathbf{1}(\mathbb{R} ; \mathbb{R})}},\left\|\tilde{\rho}_{o}\right\|_{\mathbf{L}^{\mathbf{1}}(\mathbb{R} ; \mathbb{R})}\right\}\left\|\partial_{x} f\right\|_{\mathbf{L}^{\infty}\left(\Sigma_{t} ; \mathbb{R}\right)}\left\|v^{\prime}\right\|_{\mathbf{L}^{\infty}(\mathbb{R} ; \mathbb{R})} \\
& \left.+\left\|\partial_{\rho} f\right\|_{\mathbf{L}^{\infty}\left(\Sigma_{t} ; \mathbb{R}\right)}\left(\mathcal{K}_{2} t+\operatorname{TV}\left(\rho_{o}\right)\right) e^{\mathcal{K}_{1} t}\left\|v^{\prime}\right\|_{\mathbf{L}^{\infty}(\mathbb{R} ; \mathbb{R})} \min \left\{M_{t}, \tilde{M}_{t}\right\}\right]
\end{aligned}
$$

and

$$
\begin{aligned}
& b(s) \\
= & \|f\|_{\mathbf{L}^{\infty}\left(\Sigma_{s} ; \mathbb{R}\right)} \min \left\{\|w\|_{\mathbf{W}^{1,1}(\mathbb{R} ; \mathbb{R})},\|\tilde{w}\|_{\mathbf{W}^{1,1}(\mathbb{R} ; \mathbb{R})}\right\} \\
& \times\left(\left\|v^{\prime}\right\|_{\mathbf{L}^{\infty}(\mathbb{R} ; \mathbb{R})}+\left\|v^{\prime \prime}\right\|_{\mathbf{L}^{\infty}(\mathbb{R} ; \mathbb{R})} \min \left\{\left\|\rho_{o}\right\|_{\mathbf{L}^{\mathbf{1}}(\mathbb{R} ; \mathbb{R})}\left\|\partial_{x} w\right\|_{\mathbf{L}^{\infty}(\mathbb{R} ; \mathbb{R})},\left\|\tilde{\rho}_{o}\right\|_{\mathbf{L}^{\mathbf{1}}(\mathbb{R} ; \mathbb{R})}\left\|\partial_{x} \tilde{w}\right\|_{\mathbf{L}^{\infty}(\mathbb{R} ; \mathbb{R})}\right\}\right) \\
& +\left\|\partial_{x} f\right\|_{\mathbf{L}^{\infty}\left(\Sigma_{s} ; \mathbb{R}\right)}\left\|v^{\prime}\right\|_{\mathbf{L}^{\infty}(\mathbb{R} ; \mathbb{R})} \min \left\{\|w\|_{\mathbf{L}^{1}(\mathbb{R} ; \mathbb{R})},\|\tilde{w}\|_{\mathbf{L}^{1}(\mathbb{R} ; \mathbb{R})}\right\}
\end{aligned}
$$




$$
+\left\|\partial_{\rho} f\right\|_{\mathbf{L}^{\infty}\left(\Sigma_{s} ; \mathbb{R}\right)}\left(\mathcal{K}_{2} s+\operatorname{TV}\left(\rho_{o}\right)\right) e^{\mathcal{K}_{1} s}\left\|v^{\prime}\right\|_{\mathbf{L}^{\infty}(\mathbb{R} ; \mathbb{R})} \min \left\{\|w\|_{\mathbf{L}^{\infty}(\mathbb{R} ; \mathbb{R})},\|\tilde{w}\|_{\mathbf{L}^{\infty}(\mathbb{R} ; \mathbb{R})}\right\},
$$

$\mathcal{K}_{1}$ and $\mathcal{K}_{2}$ being specified in (2.5). An application of Gronwall Lemma yields

$$
\begin{aligned}
\|\rho(t)-\tilde{\rho}(t)\|_{\mathbf{L}^{1}(\mathbb{R} ; \mathbb{R})} \leq & \left\|\rho_{o}-\tilde{\rho}_{o}\right\|_{\mathbf{L}^{1}(\mathbb{R} ; \mathbb{R})}+a(t)\|w-\tilde{w}\|_{\mathbf{W}^{1,1}(\mathbb{R} ; \mathbb{R})} \\
& +\int_{0}^{t}\left(\left\|\rho_{o}-\tilde{\rho}_{o}\right\|_{\mathbf{L}^{1}(\mathbb{R} ; \mathbb{R})}+a(s)\right) b(s) \exp \left(\int_{s}^{t} b(r) \mathrm{d} r\right) \mathrm{d} s .
\end{aligned}
$$

Since $a(s) \leq a(t)$ for any $s \in[0, t]$ and

$$
\int_{0}^{t} b(s) \exp \left(\int_{s}^{t} b(r) \mathrm{d} r\right) \mathrm{d} s=\left[-\exp \left(\int_{s}^{t} b(r) \mathrm{d} r\right)\right]_{0}^{t}=-1+\exp \left(\int_{0}^{t} b(r) \mathrm{d} r\right),
$$

we obtain

$$
\|\rho(t)-\tilde{\rho}(t)\|_{\mathbf{L}^{1}(\mathbb{R} ; \mathbb{R})} \leq\left(\left\|\rho_{o}-\tilde{\rho}_{o}\right\|_{\mathbf{L}^{1}(\mathbb{R} ; \mathbb{R})}+a(t)\|w-\tilde{w}\|_{\mathbf{W}^{1,1}(\mathbb{R} ; \mathbb{R})}\right) \exp \left(\int_{0}^{t} b(r) \mathrm{d} r\right)
$$

concluding the proof.

Remark 2. Notice that, when $t=0$, the right hand side of $(\underline{3.56})$ is equal to $\left\|\rho_{o}-\tilde{\rho}_{o}\right\|_{\mathbf{L}^{1}(\mathbb{R} ; \mathbb{R})}$, since $a(0)=0$.

Remark 3. Compare our estimate (3.56) with the one in [5, Theorem 4.1]:

$$
\|\rho(t)-\tilde{\rho}(t)\|_{\mathbf{L}^{1}(\mathbb{R} ; \mathbb{R})} \leq e^{C_{2} t}\left\|\rho_{o}-\tilde{\rho}\right\|_{\mathbf{L}^{1}(\mathbb{R} ; \mathbb{R})},
$$

where

$$
\begin{aligned}
C_{2}=\|f\|_{\mathbf{L}^{\infty}\left(\Sigma_{t} ; \mathbb{R}\right)} & \left(\left\|v^{\prime}\right\|_{\mathbf{L}^{\infty}(\mathbb{R} ; \mathbb{R})}\left\|\partial_{x} w\right\|_{\mathbf{L}^{\mathbf{1}}(\mathbb{R} ; \mathbb{R})}\right. \\
& \left.+\left\|v^{\prime \prime}\right\|_{\mathbf{L}^{\infty}(\mathbb{R} ; \mathbb{R})}\left\|\partial_{x} w\right\|_{\mathbf{L}^{\infty}(\mathbb{R} ; \mathbb{R})}\|w\|_{\mathbf{L}^{\mathbf{1}(\mathbb{R} ; \mathbb{R})}} \min \left\{\left\|\rho_{o}\right\|_{\mathbf{L}^{\mathbf{1}(\mathbb{R} ; \mathbb{R})}},\left\|\tilde{\rho}_{o}\right\|_{\mathbf{L}^{\mathbf{1}}(\mathbb{R} ; \mathbb{R})}\right\}\right) \\
+\left\|f^{\prime}\right\|_{\mathbf{L}^{\infty}\left(\Sigma_{t} ; \mathbb{R}\right)} & \operatorname{TV}(\rho(t))\left\|v^{\prime}\right\|_{\mathbf{L}^{\infty}(\mathbb{R} ; \mathbb{R})}\|w\|_{\mathbf{L}^{\infty}(\mathbb{R} ; \mathbb{R})} .
\end{aligned}
$$

The main hypotheses there are the following:

- $f(t, x, \rho)=f(\rho)$;

- $w=\tilde{w}$, thus the kernel functions are the same;

- different initial data: $\rho_{o} \neq \tilde{\rho}_{o}$.

It is immediate to see that, once the estimate for the total variation of $\rho(t)$ is inserted, the bound $C_{2}$ bears a strong resemblance with our $b(t)(3.55)$, provided the $\mathbf{L}^{\mathbf{1}}$-norm of the kernel $w$ and of its derivative are controlled by $\|w\|_{\mathbf{W}^{1,1}(\mathbb{R} ; \mathbb{R})}$. 
Remark 4. One may wonder why there is the need to exploit the doubling of variables method and to go through all the steps of the proof instead of using the ready-made estimate provided in [19, Theorem 2.5 or Proposition 2.9]. The reason lies in the coefficient $\kappa^{*}$ appearing in the estimates presented in that work. Indeed, with our notation, this coefficient reads

$$
\kappa^{*}=\left\|\partial_{\rho} \partial_{x}(f(t, x, \rho)(V(t, x)-\tilde{V}(t, x)))\right\|_{\mathbf{L}^{\infty}\left(\Sigma_{t} ; \mathbb{R}\right)} .
$$

Computing the derivatives yields

$$
\kappa^{*} \leq\left\|\partial_{x} \partial_{\rho} f\right\|_{\mathbf{L}^{\infty}\left(\Sigma_{t} ; \mathbb{R}\right)}\|V-\tilde{V}\|_{\mathbf{L}^{\infty}([0, t] \times \mathbb{R} ; \mathbb{R})}+\left\|\partial_{\rho} f\right\|_{\mathbf{L}^{\infty}\left(\Sigma_{t} ; \mathbb{R}\right)}\left\|\partial_{x} V-\partial_{x} \tilde{V}\right\|_{\mathbf{L}^{\infty}([0, t] \times \mathbb{R} ; \mathbb{R})} .
$$

Substitute now the definitions of $V$ and $\tilde{V}$, using also the estimates for (3.48) and (3.50) computed in the proof of Theorem 1] we obtain an estimate for $\kappa^{*}$ depending on the term $\|\rho-\tilde{\rho}\|_{\mathbf{L}^{\infty}\left([0, t] ; \mathbf{L}^{\mathbf{1}}(\mathbb{R} ; \mathbb{R})\right)}$. Going back to the estimate presented in [19], we see that the coefficient $\kappa^{*}$ appears in the term $e^{\kappa^{*} t}\left\|\rho_{o}-\tilde{\rho}_{o}\right\|_{\mathbf{L}^{1}(\mathbb{R} ; \mathbb{R})}$. Therefore, since the final goal is to control from above $\|\rho(t)-\tilde{\rho}(t)\|_{\mathbf{L}^{\mathbf{1}(\mathbb{R} ; \mathbb{R})}}$, we get an implicit estimate for it, which is clearly not what desired.

Proof of Theorem 2. We can apply Lemma 4 to problems (2.9) and (2.10). Let us start from the inequality $[(\underline{3.2})-(\sqrt{3.3})]$. Introduce the following notation, based on Lemma 3;

$$
\|\rho(t)\|_{\mathbf{L}^{\infty}(\mathbb{R} ; \mathbb{R})} \leq\left\|\rho_{o}\right\|_{\mathbf{L}^{\infty}(\mathbb{R} ; \mathbb{R})} e^{\mathcal{L} t} \quad\|\tilde{\rho}(t)\|_{\mathbf{L}^{\infty}(\mathbb{R} ; \mathbb{R})} \leq\left\|\rho_{o}\right\|_{\mathbf{L}^{\infty}(\mathbb{R} ; \mathbb{R})} e^{\tilde{\mathcal{L}} t} .
$$

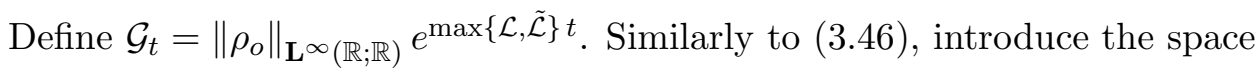

$$
\Sigma_{t}=[0, t] \times \mathbb{R} \times\left[0, \mathcal{G}_{t}\right] .
$$

Let $\tau \rightarrow 0$ in [(13.2) $-(\underline{(3.3)})]$ and recall also the assumption $\sup _{t, x}\left|\partial_{x} f(t, x, \rho)\right|<C|\rho|$ :

$$
\begin{aligned}
\|\rho(t)-\tilde{\rho}(t)\|_{\mathbf{L}^{1}(\mathbb{R} ; \mathbb{R})} \leq & \int_{0}^{t}\|f\|_{\mathbf{L}^{\infty}\left(\Sigma_{s} ; \mathbb{R}\right)} \int_{\mathbb{R}}\left|\partial_{x} V(s, x)-\partial_{x} \tilde{V}(s, x)\right| \mathrm{d} x \mathrm{~d} s \\
& +\int_{0}^{t} \int_{\mathbb{R}} C|\rho(t, x)||\tilde{V}(s, x)-V(s, x)| \mathrm{d} x \mathrm{~d} s \\
& +\int_{0}^{t}\left\|\partial_{\rho} f\right\|_{\mathbf{L}^{\infty}\left(\Sigma_{s} ; \mathbb{R}\right)} \int_{\mathbb{R}}\left|\partial_{x} \rho(s, x)\right||V(s, x)-\tilde{V}(s, x)| \mathrm{d} x \mathrm{~d} s .
\end{aligned}
$$

By the definitions of $V$ and $\tilde{V}$, compute:

$$
\begin{aligned}
& |V(s, x)-\tilde{V}(s, x)| \\
= & |v((\rho(s) * w)(x))-\tilde{v}((\tilde{\rho}(s) * w)(x))| \\
\leq & \min \left\{\left\|v^{\prime}\right\|_{\mathbf{L}^{\infty}(\mathbb{R} ; \mathbb{R})},\left\|\tilde{v}^{\prime}\right\|_{\mathbf{L}^{\infty}(\mathbb{R} ; \mathbb{R})}\right\}\|w\|_{\mathbf{L}^{\infty}(\mathbb{R} ; \mathbb{R})}\|\rho(s)-\tilde{\rho}(s)\|_{\mathbf{L}^{1}(\mathbb{R} ; \mathbb{R})}+\|v-\tilde{v}\|_{\mathbf{L}^{\infty}(\mathbb{R} ; \mathbb{R})}
\end{aligned}
$$

and

$$
\int_{\mathbb{R}}\left|\partial_{x} V(s, x)-\partial_{x} \tilde{V}(s, x)\right| \mathrm{d} x
$$




$$
\begin{aligned}
= & \int_{\mathbb{R}}\left|v^{\prime}((\rho(s) * w)(x))\left(\rho(s) * \partial_{x} w\right)(x)-\tilde{v}^{\prime}((\tilde{\rho}(s) * w)(x))\left(\tilde{\rho}(s) * \partial_{x} w\right)(x)\right| \mathrm{d} x \\
\leq & \min \left\{\left\|v^{\prime}\right\|_{\mathbf{L}^{\infty}(\mathbb{R} ; \mathbb{R})},\left\|\tilde{v}^{\prime}\right\|_{\mathbf{L}^{\infty}(\mathbb{R} ; \mathbb{R})}\right\}\left\|\partial_{x} w\right\|_{\mathbf{L}^{\mathbf{1}}(\mathbb{R} ; \mathbb{R})}\|\rho(s)-\tilde{\rho}(s)\|_{\mathbf{L}^{\mathbf{1}}(\mathbb{R} ; \mathbb{R})} \\
& +\left\|v^{\prime}-\tilde{v}^{\prime}\right\|_{\mathbf{L}^{\infty}(\mathbb{R} ; \mathbb{R})}\left\|\partial_{x} w\right\|_{\mathbf{L}^{\mathbf{1}}(\mathbb{R} ; \mathbb{R})} \min \left\{\|\rho(s)\|_{\mathbf{L}^{\mathbf{1}}(\mathbb{R} ; \mathbb{R})},\|\tilde{\rho}(s)\|_{\mathbf{L}^{\mathbf{1}}(\mathbb{R} ; \mathbb{R})}\right\} \\
\leq & \min \left\{\left\|v^{\prime}\right\|_{\mathbf{L}^{\infty}(\mathbb{R} ; \mathbb{R})},\left\|\tilde{v}^{\prime}\right\|_{\mathbf{L}^{\infty}(\mathbb{R} ; \mathbb{R})}\right\}\left\|\partial_{x} w\right\|_{\mathbf{L}^{\mathbf{1}}(\mathbb{R} ; \mathbb{R})}\|\rho(s)-\tilde{\rho}(s)\|_{\mathbf{L}^{\mathbf{1}(\mathbb{R} ; \mathbb{R})}} \\
& +\left\|v^{\prime}-\tilde{v}^{\prime}\right\|_{\mathbf{L}^{\infty}(\mathbb{R} ; \mathbb{R})}\left\|\partial_{x} w\right\|_{\mathbf{L}^{\mathbf{1}}(\mathbb{R} ; \mathbb{R})}\left\|\rho_{o}\right\|_{\mathbf{L}^{\mathbf{1}}(\mathbb{R} ; \mathbb{R})},
\end{aligned}
$$

where we exploit also Lemma 2, Therefore the inequality [(‥57) $-(\underline{3.59})]$ can be estimated as follows:

$$
\begin{aligned}
& \|\rho(t)-\tilde{\rho}(t)\|_{\mathbf{L}^{1}(\mathbb{R} ; \mathbb{R})} \\
\leq & c_{1}(t)\|v-\tilde{v}\|_{\mathbf{L}^{\infty}(\mathbb{R} ; \mathbb{R})}+c_{2}(t)\left\|v^{\prime}-\tilde{v}^{\prime}\right\|_{\mathbf{L}^{\infty}(\mathbb{R} ; \mathbb{R})}+\int_{0}^{t} c_{3}(s)\|\rho(s)-\tilde{\rho}(s)\|_{\mathbf{L}^{1}(\mathbb{R} ; \mathbb{R})} \mathrm{d} s,
\end{aligned}
$$

where, thanks to the total variation estimate provided by Proposition 1 ,

$$
\begin{aligned}
& c_{1}(t)= t\left(C\left\|\rho_{o}\right\|_{\mathbf{L}^{\mathbf{1}}(\mathbb{R} ; \mathbb{R})}+\left(\mathcal{K}_{2} t+\operatorname{TV}\left(\rho_{o}\right)\right) e^{\mathcal{K}_{1} t}\left\|\partial_{\rho} f\right\|_{\mathbf{L}^{\infty}\left(\Sigma_{t} ; \mathbb{R}\right)}\right), \\
& c_{2}(t)= t\|f\|_{\mathbf{L}^{\infty}\left(\Sigma_{t} ; \mathbb{R}\right)}\left\|\partial_{x} w\right\|_{\mathbf{L}^{\mathbf{1}}(\mathbb{R} ; \mathbb{R})}\left\|\rho_{o}\right\|_{\mathbf{L}^{\mathbf{1}}(\mathbb{R} ; \mathbb{R})}, \\
& c_{3}(s)=\|f\|_{\mathbf{L}^{\infty}\left(\Sigma_{s} ; \mathbb{R}\right)} \min \left\{\left\|v^{\prime}\right\|_{\mathbf{L}^{\infty}(\mathbb{R} ; \mathbb{R})},\left\|\tilde{v}^{\prime}\right\|_{\mathbf{L}^{\infty}(\mathbb{R} ; \mathbb{R})}\right\}\left\|\partial_{x} w\right\|_{\mathbf{L}^{\mathbf{1}}(\mathbb{R} ; \mathbb{R})} \\
&+\left(C\left\|\rho_{o}\right\|_{\mathbf{L}^{\mathbf{1}(\mathbb{R} ; \mathbb{R})}}+\left(\mathcal{K}_{2} s+\operatorname{TV}\left(\rho_{o}\right)\right) e^{\mathcal{K}_{1} s}\left\|\partial_{\rho} f\right\|_{\mathbf{L}^{\infty}\left(\Sigma_{s} ; \mathbb{R}\right)}\right) \\
& \quad \times \min \left\{\left\|v^{\prime}\right\|_{\mathbf{L}^{\infty}(\mathbb{R} ; \mathbb{R})},\left\|\tilde{v}^{\prime}\right\|_{\mathbf{L}^{\infty}(\mathbb{R} ; \mathbb{R})}\right\}\|w\|_{\mathbf{L}^{\infty}(\mathbb{R} ; \mathbb{R})},
\end{aligned}
$$

$\mathcal{K}_{1}$ and $\mathcal{K}_{2}$ being specified in (2.5). An application of Gronwall Lemma yields

$$
\|\rho(t)-\tilde{\rho}(t)\|_{\mathbf{L}^{\mathbf{1}(\mathbb{R} ; \mathbb{R})}} \leq\left(c_{1}(t)\|v-\tilde{v}\|_{\mathbf{L}^{\infty}(\mathbb{R} ; \mathbb{R})}+c_{2}(t)\left\|v^{\prime}-\tilde{v}^{\prime}\right\|_{\mathbf{L}^{\infty}(\mathbb{R} ; \mathbb{R})}\right) \exp \left(\int_{0}^{t} c_{3}(s) \mathrm{d} s\right),
$$

concluding the proof.

\section{Numerical Integrations}

In this section, we investigate the dependence of solutions to (1.1) on the kernel and the velocity function via numerical integrations. To this end, we discretize (1.1) on a fixed grid given by the cells interfaces $x_{j+\frac{1}{2}}=j \Delta x$ and the cells centres $x_{j}=\left(j-\frac{1}{2}\right) \Delta x$ for $j \in \mathbb{Z}$, taking a space step $\Delta x$ and a time step $\Delta t$, so that $t^{n}=n \Delta t$ is the time mesh. The Lax-Friedrichs flux adapted to (1.1) is given by

$$
F_{j+1 / 2}^{n}:=\frac{1}{2}\left(f\left(t^{n}, x_{j}, \rho_{j}^{n}\right) v\left(R_{j}^{n}\right)+f\left(t^{n}, x_{j+1}, \rho_{j+1}^{n}\right) v\left(R_{j+1}^{n}\right)\right)-\frac{\alpha}{2}\left(\rho_{j+1}^{n}-\rho_{j}^{n}\right)
$$

where $\alpha \geq 0$ is the viscosity coefficient and $R_{j}^{n}:=\Delta x \sum_{k \in \mathbb{Z}} \rho_{j+k}^{n} w_{\eta}^{k}$, denoting $w_{\eta}^{k}:=w_{\eta}(k \Delta x)$ for $k \in \mathbb{Z}$. In this way we have the finite volume scheme

$$
\rho_{j}^{n+1}=\rho_{j}^{n}-\lambda\left[F_{j+1 / 2}^{n}-F_{j-1 / 2}^{n}\right],
$$


with $\lambda=\Delta t / \Delta x$. A rigorous study of the convergence of Lax-Friedrichs type schemes for non-local conservation laws has been carried out in [1, 4, 6]. Here we limit the study to the derivation of sufficient conditions ensuring that the above discretization (4.1)-(4.2) is positivity preserving.

Lemma 5. For any $T>0$, under the CFL conditions

$$
\begin{gathered}
\lambda\left(\alpha+\left(C \Delta x+2\left\|\partial_{\rho} f\right\|_{\mathbf{L}^{\infty}\left(\Sigma_{T} ; \mathbb{R}\right)}\right)\|v\|_{\mathbf{L}^{\infty}(\mathbb{R} ; \mathbb{R})}\right)<1, \\
\alpha \geq\left\|\partial_{\rho} f\right\|_{\mathbf{L}^{\infty}\left(\Sigma_{T} ; \mathbb{R}\right)}\|v\|_{\mathbf{L}^{\infty}(\mathbb{R} ; \mathbb{R})},
\end{gathered}
$$

the scheme (4.1)-(4.2) is positivity preserving on $[0, T] \times \mathbb{R}$.

Proof. Let us assume that $\rho_{j}^{n} \geq 0$ for all $j \in \mathbb{Z}$. It suffices to prove that $\rho_{j}^{n+1}$ in (4.2) is non-negative. For the sake of simplicity, in the following we omit the dependence on $n$ and introduce the notation $f_{i}\left(\rho_{j}\right)=f\left(t^{n}, x_{i}, \rho_{j}\right)$ and $v_{j}=v\left(R_{j}^{n}\right)$. Compute

$$
\begin{aligned}
\rho_{j}^{n+1}= & \rho_{j}+\frac{\lambda \alpha}{2}\left(\rho_{j+1}-2 \rho_{j}+\rho_{j-1}\right)-\frac{\lambda}{2}\left[f_{j+1}\left(\rho_{j+1}\right) v_{j+1}-f_{j-1}\left(\rho_{j-1}\right) v_{j-1}\right] \\
= & \rho_{j}(1-\lambda \alpha)+\frac{\lambda \alpha}{2}\left(\rho_{j+1}+\rho_{j-1}\right) \\
& -\frac{\lambda}{2}\left[\left(f_{j+1}\left(\rho_{j+1}\right)-f_{j+1}\left(\rho_{j}\right)\right) v_{j+1}+\left(f_{j-1}\left(\rho_{j}\right)-f_{j-1}\left(\rho_{j-1}\right)\right) v_{j-1}\right. \\
& \left.\quad+\left(f_{j+1}\left(\rho_{j}\right)-f_{j-1}\left(\rho_{j}\right)\right) v_{j+1}+f_{j-1}\left(\rho_{j}\right)\left(v_{j+1}-v_{j-1}\right)\right] \\
= & \rho_{j}\left(1-\lambda \alpha+\frac{\lambda}{2} \frac{f_{j+1}\left(\rho_{j+1}\right)-f_{j+1}\left(\rho_{j}\right)}{\rho_{j+1}-\rho_{j}} v_{j+1}-\frac{\lambda}{2} \frac{f_{j-1}\left(\rho_{j}\right)-f_{j-1}\left(\rho_{j-1}\right)}{\rho_{j}-\rho_{j-1}} v_{j-1}\right) \\
& +\rho_{j+1}\left(\frac{\lambda \alpha}{2}-\frac{\lambda}{2} \frac{f_{j+1}\left(\rho_{j+1}\right)-f_{j+1}\left(\rho_{j}\right)}{\rho_{j+1}-\rho_{j}} v_{j+1}\right) \\
& +\rho_{j-1}\left(\frac{\lambda \alpha}{2}+\frac{\lambda}{2} \frac{f_{j-1}\left(\rho_{j}\right)-f_{j-1}\left(\rho_{j-1}\right)}{\rho_{j}-\rho_{j-1}} v_{j-1}\right) \\
& -\frac{\lambda}{2} v_{j+1}\left(f_{j+1}\left(\rho_{j}\right)-f_{j-1}\left(\rho_{j}\right)\right)-\frac{\lambda}{2} f_{j-1}\left(\rho_{j}\right)\left(v_{j+1}-v_{j-1}\right) .
\end{aligned}
$$

Observe that, thanks to the assumption (4.4) on $\alpha$,

$$
\begin{aligned}
& \alpha+\frac{f_{j-1}\left(\rho_{j}\right)-f_{j-1}\left(\rho_{j-1}\right)}{\rho_{j}-\rho_{j-1}} v_{j-1}=\alpha+\partial_{\rho} f_{j-1}\left(\zeta_{j-1 / 2}\right) v_{j-1} \geq \alpha-\left\|\partial_{\rho} f\right\|_{\mathbf{L}^{\infty}\left(\Sigma_{T} ; \mathbb{R}\right)}\|v\|_{\mathbf{L}^{\infty}(\mathbb{R} ; \mathbb{R})} \geq 0, \\
& \alpha-\frac{f_{j+1}\left(\rho_{j+1}\right)-f_{j+1}\left(\rho_{j}\right)}{\rho_{j+1}-\rho_{j}} v_{j+1}=\alpha-\partial_{\rho} f_{j+1}\left(\zeta_{j+1 / 2}\right) v_{j+1} \geq \alpha-\left\|\partial_{\rho} f\right\|_{\mathbf{L}^{\infty}\left(\Sigma_{T} ; \mathbb{R}\right)}\|v\|_{\mathbf{L}^{\infty}(\mathbb{R} ; \mathbb{R})} \geq 0 .
\end{aligned}
$$

Moreover,

$$
v_{j+1}\left(f_{j+1}\left(\rho_{j}\right)-f_{j-1}\left(\rho_{j}\right)\right) \leq 2 C\|v\|_{\mathbf{L}^{\infty}(\mathbb{R} ; \mathbb{R})} \Delta x \rho_{j}
$$

and

$$
f_{j-1}\left(\rho_{j}\right)\left(v_{j+1}-v_{j-1}\right) \leq 2\left\|\partial_{\rho} f\right\|_{\mathbf{L}^{\infty}\left(\Sigma_{T} ; \mathbb{R}\right)}\|v\|_{\mathbf{L}^{\infty}(\mathbb{R} ; \mathbb{R})} \rho_{j} .
$$


Hence,

$$
\begin{aligned}
\rho_{j} & \left(1-\lambda \alpha+\frac{\lambda}{2} \frac{f_{j+1}\left(\rho_{j+1}\right)-f_{j+1}\left(\rho_{j}\right)}{\rho_{j+1}-\rho_{j}} v_{j+1}-\frac{\lambda}{2} \frac{f_{j-1}\left(\rho_{j}\right)-f_{j-1}\left(\rho_{j-1}\right)}{\rho_{j}-\rho_{j-1}} v_{j-1}\right) \\
& -\frac{\lambda}{2} v_{j+1}\left(f_{j+1}\left(\rho_{j}\right)-f_{j-1}\left(\rho_{j}\right)\right)-\frac{\lambda}{2} f_{j-1}\left(\rho_{j}\right)\left(v_{j+1}-v_{j-1}\right) \\
\geq & \rho_{j}\left(1-\lambda \alpha-2 \lambda\left\|\partial_{\rho} f\right\|_{\mathbf{L}^{\infty}\left(\Sigma_{T} ; \mathbb{R}\right)}\|v\|_{\mathbf{L}^{\infty}(\mathbb{R} ; \mathbb{R})}-\lambda C\|v\|_{\mathbf{L}^{\infty}(\mathbb{R} ; \mathbb{R})} \Delta x\right) \geq 0,
\end{aligned}
$$

by the CFL condition (4.3).

Fix $T=0.5$. Let us now consider the following problem:

$$
\left\{\begin{array}{l}
\left.\partial_{t} \rho+\partial_{x}\left(f(t, x, \rho) v\left(w_{\eta, \delta} * \rho\right)\right)=0, \quad t \in[0, T], x \in\right]-1,1[, \\
\rho(0, x)=0.6
\end{array}\right.
$$

with periodic boundary conditions at $x= \pm 1$ and

$$
\begin{array}{lr}
f(t, x, \rho)=V_{\max }(t, x) \rho(1-\rho), & \rho \in[0,1], \\
v(\rho)=(1-\rho)^{m-1}(1+\rho)^{m}, & m \in \mathbb{N}, \\
w_{\eta, \delta}(x)=\frac{1}{\eta^{6}} \frac{16}{5 \pi}\left(\eta^{2}-(x-\delta)^{2}\right)^{\frac{5}{2}} \chi_{[-\eta+\delta, \eta+\delta]}, & \eta \in] 0,1], \delta \in[-\eta, \eta] .
\end{array}
$$

In (4.6), $V_{\max }(t, x)$ is given by the convolution between the gaussian kernel $g(x)=\frac{1}{\sigma \sqrt{2 \pi}} e^{-\frac{1}{2}\left(\frac{x}{\sigma}\right)^{2}}$ with $\sigma=10$ and the following piece-wise constant function:

$$
\varphi(t, x)= \begin{cases}7 & \text { if } x \in]-1,-1 / 3] \cup] 1 / 3,1], \\ 3 & \text { if } x \in]-1 / 3,1 / 3], t \in[0,1 / 6] \cup] 1 / 3,1 / 2], \\ 1.5 & \text { if } x \in]-1 / 3,1 / 3], t \in] 1 / 6,1 / 3]\end{cases}
$$

see Figure 1. In (4.8), the parameter $\eta$ represents the radius of the support of the kernel function $w_{\eta, \delta}$, while $\delta$ is the point at which the maximum is attained.

The above equations (4.5)-(4.8) describe the traffic flow on a circular road with variable speed limit in space and time, starting from a constant initial density $\rho_{o} \equiv 0.6$ (for simplicity, the maximal density is here normalised to 1 ).

As a metric of traffic congestion, we consider the two following functionals [9, 10, 13]:

$$
\begin{gathered}
J(T)=\int_{0}^{T} \mathrm{~d}\left|\partial_{x} \rho(t, \cdot)\right| \mathrm{d} t, \\
\Psi(T ; a, b)=\int_{0}^{T} \int_{a}^{b} \varphi(\rho(t, x)) \mathrm{d} x \mathrm{~d} t,
\end{gathered}
$$

where

$$
\varphi(r)= \begin{cases}0 & r<0.75 \\ 10 r-7.5 & 0.75 \leq r \leq 0.85 \\ 1 & 0.85<r \leq 1\end{cases}
$$




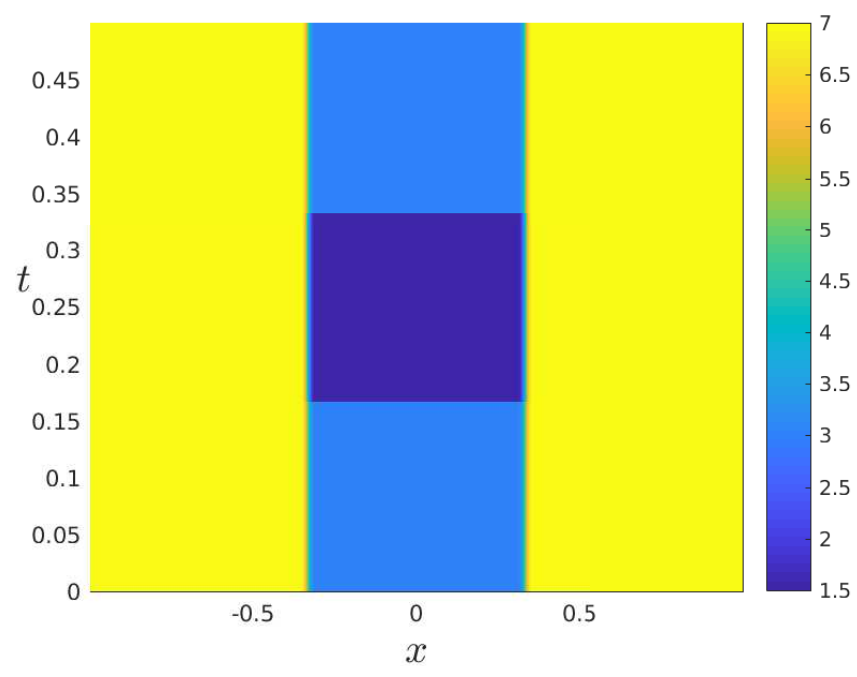

Figure 1: 2D plot of the function $V_{\max }(t, x)$

The functional $J$ defined in (4.9) measures the integral with respect to time of the spatial total variation of the traffic density. The results of Theorems 1 and 2 apply to the present setting and ensure the continuous dependence of $J$ on the parameters $m, \eta$ and $\delta$. Indeed, the map $\delta \rightarrow w_{\eta, \delta}$ is Lipschitz continuous with respect to the $\mathbf{W}^{1,1}$ distance, the map $\eta \rightarrow w_{\eta, \delta}$ is continuous with respect to the $\mathbf{W}^{1,1}$ distance and the map $m \rightarrow v$ is continuous with respect to the $\mathbf{W}^{1, \infty}$ distance. Theorem 1 then ensures that the map $w_{\eta, \delta} \rightarrow \rho$, where $\rho$ solves (4.5)-(4.8), is continuous with respect to the $\mathbf{W}^{1,1}$ distance, while Theorem 2 ensures the continuity of the map $v \rightarrow \rho$. Finally, the map $\rho \rightarrow J$ is lower semicontinuous, as showed in [10, Lemma 2.1]. Therefore, any minimising sequence of solutions converges, guaranteeing the existence of optimal choices of the parameters $\eta, \delta$ and $m$.

The functional $\Psi$ in (4.10) was introduced in 13 , and it is obviously continuous with respect to $\rho$ in the $\mathbf{L}^{\mathbf{1}}$-distance. It measures the queue of the solution in the space interval $[a, b]$, which is chosen equal to $[-4 / 5,-1 / 3]$ in the numerical simulations below.

For the tests, we fix the space discretization mesh to $\Delta x=0.001$. Figures 23 show the values of the functionals $J$ and $\psi$ when we vary the value of one of the parameters $\eta, \delta$ and $m$, keeping the other fixed. In particular, the functionals are evaluated on the following grids:

$$
\eta=0.1: 0.1: 1, \quad \delta=-0.1: 0.02: 0.1, \quad m=1: 1: 10 .
$$

We observe that the functionals are in general not monotone and display some extrema in the considered intervals. Figures [5, 6] and 7 show the behaviour of the solutions corresponding to some of these extremal values. More precisely, Figures $5 \mathrm{a}, 5 \mathrm{~b}$ and $5 \mathrm{c}$ show the solutions corresponding to $\eta=0.2,0.5,1$ for $m=3$ and centered kernel $(\delta=0)$. In particular, the solutions displayed in $5 \mathrm{a}$ and $5 \mathrm{c}$ correspond to the minimum and maximum values of the functional $J$ (4.9) for $\eta \in[0.1,1]$ (see Figure 2, left). Figure 6a shows the solution obtained for $\delta=-0.04$ (and $m=3, \eta=0.1$ ) and corresponding to the point of minimum of both $J$ and $\Psi$ functionals, while Figures $6 \mathrm{~b}$ and $6 \mathrm{c}$ correspond to the points of maximum of the functionals $J$ and $\Psi$, respectively (see Figure 3). Finally, in Figures $7 \mathrm{a}$ and $7 \mathrm{~b}$ we give the solutions corresponding to the maximum and minimum points of the functional $J$ for $m \in\{1, \ldots, 10\}$ for $\eta=0.1$ and $\delta=0$ (see Figure 4). 

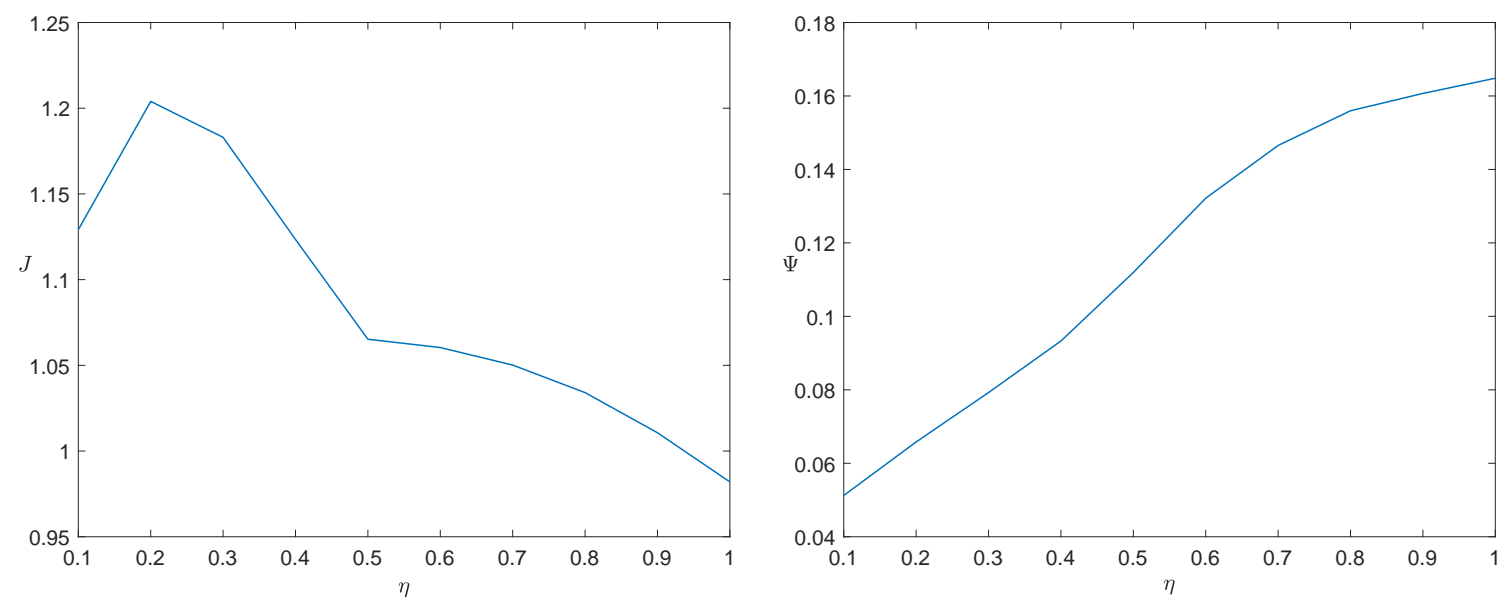

Figure 2: Functionals J (4.9) (left) and $\Psi$ (4.10) (right) with $m=3, \delta=0$ and $\eta \in[0.1,1]$.
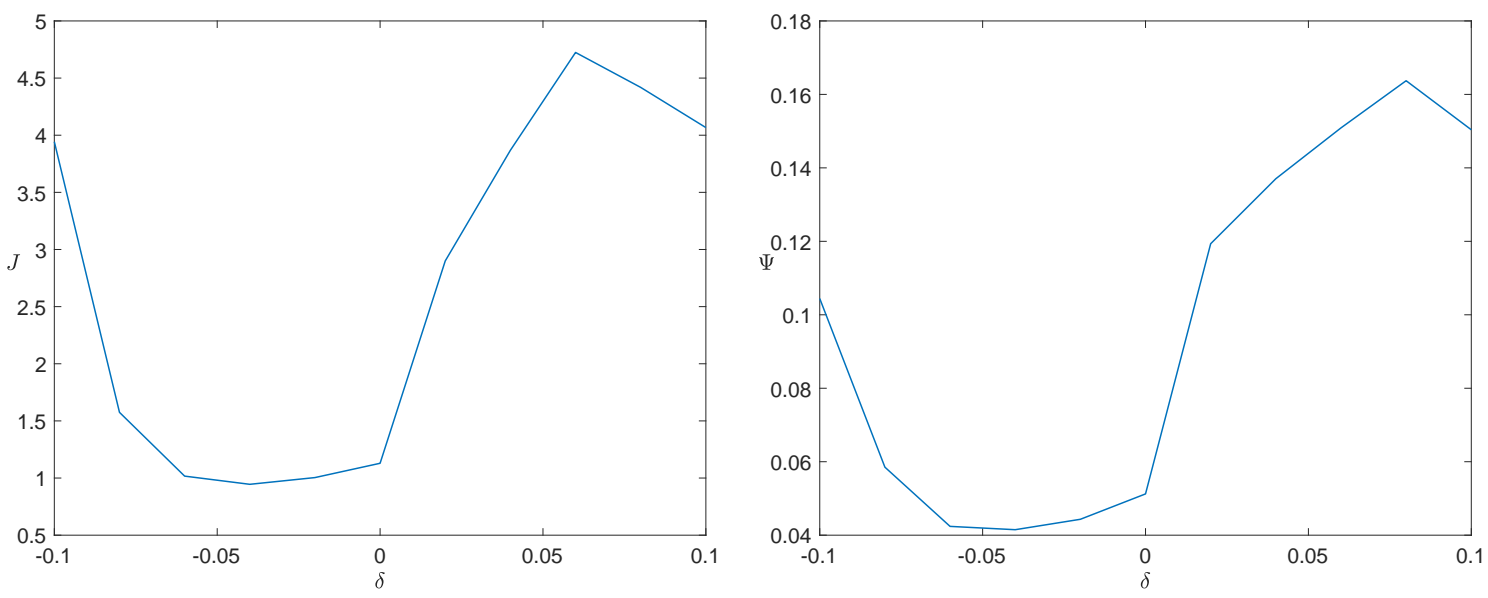

Figure 3: Functionals $J$ (4.9) (left) and $\Psi$ (4.10) (right) with $\eta=0.1, m=3$ and $\delta \in[-\eta, \eta]$.
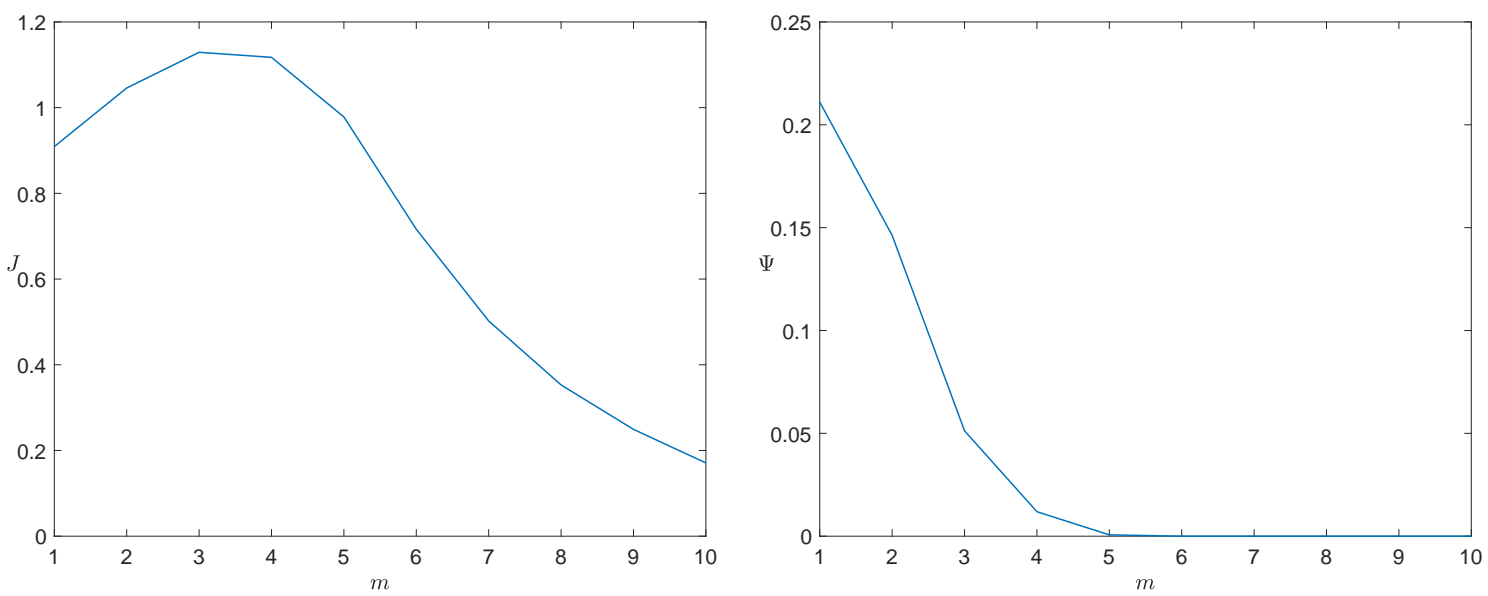

Figure 4: Functionals $J$ (4.9) (left) and $\Psi$ (4.10) (right) with $\eta=0.1, \delta=0$ and $m \in[1,10]$. 


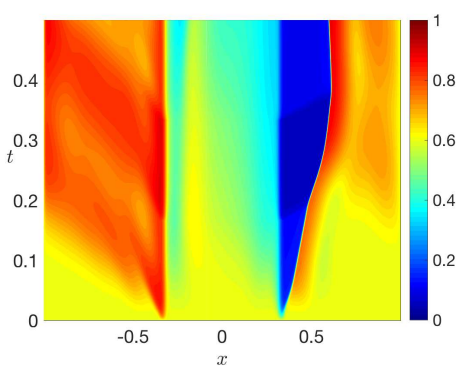

(a)

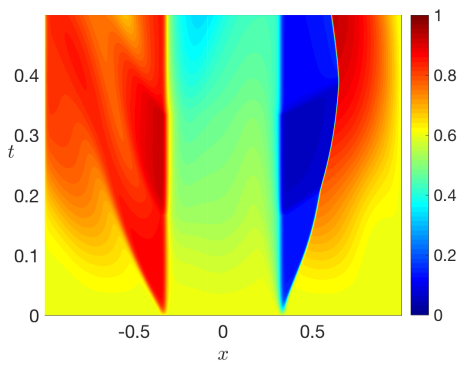

(b)

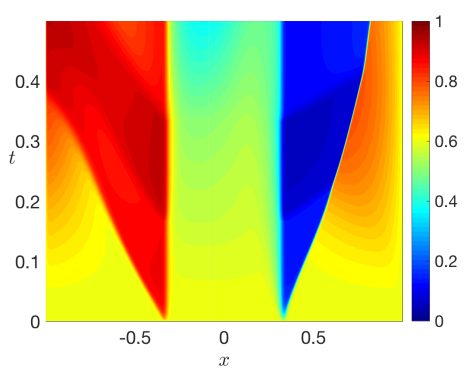

(c)

Figure 5: $(t, x)$-plots of the solution to (4.5)-(4.8), for $m=3$ and $\delta=0$, and, from the left, $\eta=0.2,0.5,1$.

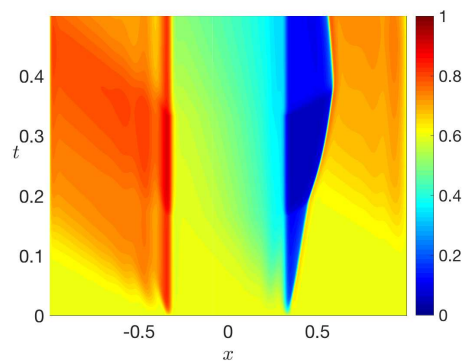

(a)

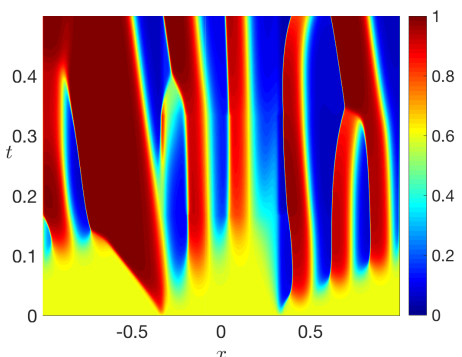

(b)

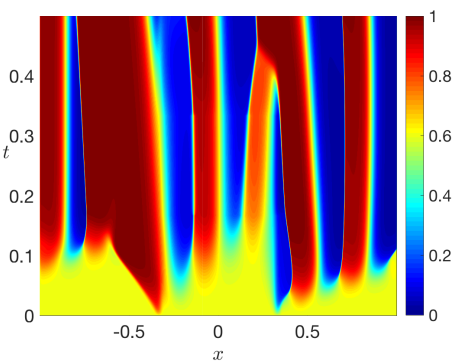

(c)

Figure 6: $(t, x)$-plots of the solution to (4.5)-(4.8), for $m=3$ and $\eta=0.1$, and, from the left, $\delta=-0.04,0.06,0.08$.

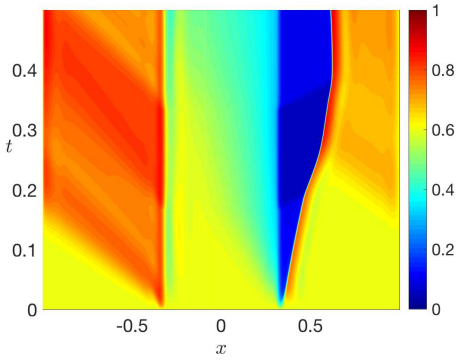

(a)

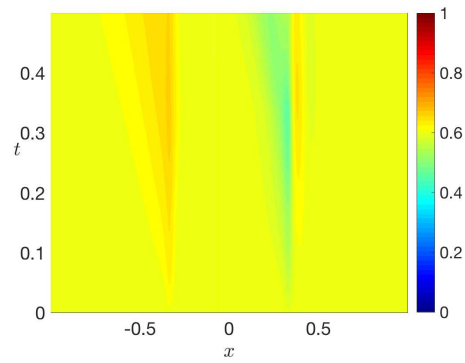

(b)

Figure 7: $(t, x)$-plots of the solution to (4.5)-(4.8), for $\eta=0.1, \delta=0$, and $m=3$ on the left, $m=10$ on the right.

\section{References}

[1] A. Aggarwal, R. M. Colombo, and P. Goatin. Nonlocal systems of conservation laws in several space dimensions. SIAM J. Numer. Anal., 53(2):963-983, 2015.

[2] D. Amadori, S.-Y. Ha, and J. Park. On the global well-posedness of BV weak solutions to the Kuramoto-Sakaguchi equation. J. Differential Equations, 262(2):978-1022, 2017.

[3] D. Amadori and W. Shen. An integro-differential conservation law arising in a model of granular flow. J. Hyperbolic Differ. Equ., 9(1):105-131, 2012. 
[4] P. Amorim, R. Colombo, and A. Teixeira. On the numerical integration of scalar nonlocal conservation laws. ESAIM M2AN, 49(1):19-37, 2015.

[5] F. Betancourt, R. Bürger, K. H. Karlsen, and E. M. Tory. On nonlocal conservation laws modelling sedimentation. Nonlinearity, 24(3):855-885, 2011.

[6] S. Blandin and P. Goatin. Well-posedness of a conservation law with non-local flux arising in traffic flow modeling. Numer. Math., 132(2):217-241, 2016.

[7] F. A. Chiarello and P. Goatin. Global entropy weak solutions for general non-local traffic flow models with anisotropic kernel. ESAIM Math. Model. Numer. Anal., to appear.

[8] R. M. Colombo, M. Garavello, and M. Lécureux-Mercier. A class of nonlocal models for pedestrian traffic. Mathematical Models and Methods in Applied Sciences, 22(04):1150023, 2012.

[9] R. M. Colombo, P. Goatin, and M. D. Rosini. On the modelling and management of traffic. ESAIM Math. Model. Numer. Anal., 45(5):853-872, 2011.

[10] R. M. Colombo and A. Groli. Minimising stop and go waves to optimise traffic flow. Appl. Math. Lett., 17(6):697-701, 2004.

[11] R. M. Colombo, M. Mercier, and M. D. Rosini. Stability and total variation estimates on general scalar balance laws. Commun. Math. Sci., 7(1):37-65, 2009.

[12] R. M. Colombo and E. Rossi. Rigorous estimates on balance laws in bounded domains. Acta Math. Sci. Ser. B Engl. Ed., 35(4):906-944, 2015.

[13] R. M. Colombo and E. Rossi. IBVPs for scalar conservation laws with time discontinuous fluxes. Mathematical Methods in the Applied Sciences, To appear.

[14] S. Göttlich, S. Hoher, P. Schindler, V. Schleper, and A. Verl. Modeling, simulation and validation of material flow on conveyor belts. Applied Mathematical Modelling, 38(13):3295 - 3313, 2014.

[15] M. Gröschel, A. Keimer, G. Leugering, and Z. Wang. Regularity theory and adjoint-based optimality conditions for a nonlinear transport equation with nonlocal velocity. SIAM J. Control Optim., 52(4):2141-2163, 2014.

[16] K. H. Karlsen and N. H. Risebro. On the uniqueness and stability of entropy solutions of nonlinear degenerate parabolic equations with rough coefficients. Discrete Contin. Dyn. Syst., 9(5):1081$1104,2003$.

[17] A. Keimer and L. Pflug. Existence, uniqueness and regularity results on nonlocal balance laws. J. Differential Equations, 263(7):4023-4069, 2017.

[18] S. N. Kružkov. First order quasilinear equations with several independent variables. Mat. Sb. (N.S.), 81 (123):228-255, 1970.

[19] M. Lécureux-Mercier. Improved stability estimates on general scalar balance laws. ArXiv e-prints, July 2013.

[20] B. Perthame. Transport equations in biology. Frontiers in Mathematics. Birkhäuser Verlag, Basel, 2007.

[21] A. Sopasakis and M. A. Katsoulakis. Stochastic modeling and simulation of traffic flow: asymmetric single exclusion process with Arrhenius look-ahead dynamics. SIAM J. Appl. Math., 66(3):921-944 (electronic), 2006. 